\title{
Effects of Mindfulness-Based Parallel-Group Interventions on Family Functioning and Child and Parent Mental Health: a Systematic Review and Meta-analysis
}

\author{
Qian-Wen Xie ${ }^{1,2} \cdot$ Xiaolu Dai $^{3} \cdot$ Renhui Lyu ${ }^{3} \cdot$ Shuang $\mathrm{Lu}^{3,4}$ (1)
}

Accepted: 12 August 2021 / Published online: 24 August 2021

(c) The Author(s) 2021

\begin{abstract}
Objectives Involving parents and children in mindfulness-based interventions may holistically benefit family well-being. This meta-analysis systematically reviews and synthesizes the effects of mindfulness-based parallel-group (MBPG) interventions, which simultaneously involve parent and child, on family functioning, and the mental health of parents and children. Methods A total of 20 relevant studies were identified from 14 databases. The overall intervention effect size was estimated by pooled standardized mean difference. Moderator analyses were performed to explain the variability in intervention effects. Risk of bias and publication bias were also assessed.

Results MBPG interventions showed minor-to-small positive effects on family functioning ( $d=0.182,95 \%$ CI [0.045, $0.319])$, parental mental health $(d=0.238,95 \%$ CI [0.110, 0.365]), and child mental health $(d=0.325,95 \%$ CI [0.137, 0.513$])$. The effects of MBPG interventions on child mental health varied significantly by child age, child gender, recruitment setting, type of parent group, other activities in child group, other activities in parent group, and study design.

Conclusions MBPG interventions show promising effects in improving mental health of both parents and children as well as in improving overall family functioning. However, significant variations exist in characteristics of participants, interventions, and study designs. Given the limited evidence currently available, more studies are needed to assess the determinants of effectiveness in MBPG interventions.
\end{abstract}

Protocol Registration: PROSPERO \#CRD42020164927

Keywords Mindfulness $\cdot$ Family functioning $\cdot$ Mental health $\cdot$ Parallel group $\cdot$ Children $\cdot$ Meta-analysis

The family is an interdependent system (Bowen, 1966), within which the well-being of an individual member influences other members. Given this, mindfulness-based interventions (MBIs) incorporating mindfulness components

Shuang Lu

shuanglu@hku.hk

1 School of Public Affairs, Zhejiang University, Zijin'gang Campus, R934, SPA Bldg, 866 Yuhangtang Rd,

Hangzhou 310058, China

2 Center of Social Welfare and Governance, Zhejiang University, Zijin'gang Campus, R934, SPA Bldg, 866 Yuhangtang Rd, Hangzhou 310058, China

3 Department of Social Work and Social Administration, The University of Hong Kong, Centennial Campus, Jockey Club Tower, 5/F, Pokfulam, Hong Kong

4 Center on Behavioral Health, The University of Hong Kong, 2/F, 5 Sassoon Road, Pokfulam, Hong Kong into both parent and child activities have been increasingly provided within the family context in recent years (e.g., de Bruin et al., 2015). However, existing research focused primarily on either the effects of MBIs designed for parents or MBIs designed for youth. The overall effects of MBIs targeting the entire family remain unclear to date.

Extensive literature has supported the positive effects of MBIs targeting parents. For example, in a review of seven randomized controlled trials (RCTs) of parenting programs that either implicitly or explicitly involved mindfulness components, Townshend et al. (2016) found that most of the RCTs reported small-to-moderate effects in reducing parenting stress and improving parental emotional awareness. In a meta-analysis of MBIs for parents (Burgdorf et al., 2019), a combination of 19 studies (including 18 non-controlled studies) showed a small within-group effect of MBIs in reducing parenting stress after the intervention $(g=0.34)$; by combining five controlled studies, MBIs showed a 
small-to-moderate effect $(g=0.44)$ in reducing parenting stress compared with the control groups. A recent review of 13 parenting interventions that focused on self-compassion, most of which were MBIs, found small reductions in parent depression $(g=0.425)$, anxiety $(g=0.377)$, and stress ( $g=0.363$; effect sizes reversed for negative outcomes) based on within-group pre-posttest analyses (Jefferson et al., 2020).

Moreover, previous research found that MBIs for parents could also benefit their children. For instance, Burgdorf et al. (2019) suggested that MBIs for parents also improved child psychological outcomes, with an overall positive effect $(g=0.27)$ immediately after intervention and a sustained, increased effect at 2-month follow-up $(g=0.35)$. This positive effect remained even when children themselves were not involved in the intervention $(g=0.26)$. Similarly, Townshend et al. (2016) suggested that mindful parenting programs appeared to reduce externalizing disorder-related symptoms in preschoolers. Youth could also benefit from MBIs specifically designed for them. For instance, Zenner et al. (2014) reviewed 24 school-based MBIs delivered to children and found positive effects on child overall psychological outcomes $(g=0.4)$, and the positive effects exhibited in multiple developmental domains, such as cognitive performance and resilience to stress. Two other systematic reviews also supported the feasibility and acceptability of MBIs among adolescents with mental health conditions (Kostova et al., 2019) and youth in school settings (Felver et al., 2016).

Recently, research is emerging around mindfulness-based parallel-group (MBPG) interventions, which refer to mindfulness-based interventions simultaneously delivered to parents and children in separate groups. Preliminary evidence of MBPG interventions has yielded encouraging results in improving holistic family well-being. For parents, MBPG interventions were associated with reduced parenting stress (Haydicky et al., 2015; Lo et al., 2019) and enhanced parent psychological well-being (Lo et al., 2017). For children, MBPG interventions showed benefits such as enhanced child attention, self-regulation (Lo et al., 2019), improvements in autism (Salem-Guirgis et al., 2019), attention deficit/hyperactivity disorder (ADHD; Haydicky et al., 2015), depression (Racey et al., 2018), and anxiety (Hancock et al., 2018) symptoms. MBPG interventions were also found to reduce parent-child dysfunctional interactions in economically disadvantaged families (Lo et al., 2019). However, results on the effects of MBPG showed inconsistent directions. For example, a single-group study among 11 children with ADHD and their parents showed that parenting stress became worse at posttest than baseline, and no statistically significant changes were found in dysfunctional parenting discipline or mindful parenting (Zhang et al., 2017). Another study of preschoolers and their parents showed that MBPG had negative effect on parental emotion regulation but positive effect on parental psychological well-being (Jackman et al., 2019). There is a lack of synthesized investigation of the effectiveness of MBPG interventions that simultaneously target both the parent and the child, two fundamental components of a family system. Additionally, existing MBPG interventions involve a diversity of sampling criteria, approaches, duration, components, and structure, necessitating a systematic analysis of how these factors affect intervention effectiveness.

This study aimed to systematically review the available evidence and explore the effectiveness of MBPG interventions on the mental health of parents and children as well as overall family functioning. Mental health, a key well-being indicator of family members, refers to "a state of well-being in which the individual realizes his or her own abilities, can cope with the normal stresses of life, can work productively and fruitfully and is able to make a contribution to his or her community" (World Health Organization [WHO], 2013, p. 3). Mental health not only includes a negative dimension that concerns mental disorders and symptoms; it also involves a positive dimension that comprises positive affect, subjective well-being, and the ability to cope with adversity (European Commission, 1998; WHO, 2013). More recently, mental health is conceptualized as "a dynamic state of internal equilibrium which enables individuals to use their abilities in harmony with universal values of society" (Galderisi et al., 2015, p. 231-232). Specifically, it is considered to contain the following domains: basic cognitive and social skills, emotion regulation and empathy, flexibility, ability to cope with adversity, and harmonious relationships between the body and the mind (Galderisi et al., 2015). Family functioning is defined as "the roles that family members play, and the attitudes and behaviors they exhibit in their relationships with each other" (DeFrain et al., 2009, p. 622-623). Previous research has suggested not only the interrelationships between parental mental health and child mental health (Lohaus et al., 2017; Manning \& Gregoire, 2006), but also the close relations between the mental health of family members and overall family functioning (Goldberg \& Carlson, 2014; Wang \& Crane, 2001; Wang \& Zhou, 2015). Two research questions underpin the current systematic review and meta-analysis: (1) do MBPG interventions positively affect three interrelated outcome domains (family functioning, parental mental health, and child mental health) and (2) to what extent are these intervention effects moderated by the characteristics of participants, interventions, and studies?

\section{Method}

\section{Protocol and Registration}

This study followed the systematic review protocol PROSPERO \#CRD42020164927 (Xie et al., 2020). This review 
was conducted and reported according to the Preferred Reporting Items for Systematic Reviews and Meta-Analyses diagnostic test accuracy (PRISMA-DTA) guideline (McInnes et al., 2018).

\section{Eligibility Criteria}

English-language published journal articles and unpublished theses were included in the systematic review if they (a) estimated the effects of an MBPG intervention simultaneously providing to children or adolescents (mean age $\leq 18$ years) and their parents or caregivers in parallel group format; (b) contained outcome variables that were measures of family functioning, parental mental health, or child mental health; and (c) provided sufficient quantitative data to calculate effect sizes. We included only published journal articles if researchers reported the same data in unpublished dissertations. Books, magazines, conference abstracts, and review articles were excluded.

\section{Information Sources}

We systematically searched the following 14 electronic databases for eligible sources: British Nursing Index (from 1994), CINAHL Plus (from 1937), EMBASE (from 1974), ERIC (from 1966), Family \& Society Studies Worldwide (from 1970), MEDLINE (from 1946), ProQuest Dissertations \& Theses databases (from 1743), PsycINFO (from 1806), PubMed (from 1997), Social Work Abstracts (from 1968), Sociological Abstracts (from 1952), The Cochrane Library (from 1996), Web of Science (from 1990), and Scopus (from 2004).

\section{Search}

We conducted an initial search of above electronic databases up to September 2019 and a second-round search in April 2020. Four sets of keywords were used in combination and modified according to the requirements of the electronic databases: (1) mindfulness (Mindful*); (2) family (parent* OR mother OR father OR caregiver* OR carer* OR family OR home); (3) children or adolescents (child* OR boys OR girls OR juvenil* OR minors OR adolesc* OR preadolesc* OR pre-adolesc* OR pre-school OR preschool OR paediatric* OR pediatric* OR pubescen* OR puberty OR school* OR campus OR teen* OR young OR youth*); and (4) intervention (random* OR experiment* OR RCT OR intervention OR group OR program OR training OR therapy OR trial). We also examined the bibliographies of included studies and consulted experts in the field of mindfulness for further references to relevant studies. All records were incorporated into the current study.

\section{Study Selection}

All records were exported to EndNote software for the management of studies and elimination of duplicates. Two review authors (AU2 and AU3) independently screened titles, abstracts, and full texts according to the selection criteria. Differences were resolved in follow-up meetings between review authors.

\section{Data Collection Process}

Using a pre-piloted, standardized coding scheme, information on the characteristics of participants, interventions, and studies was independently extracted by two review authors (AU2 and AU3). All coding inconsistencies were resolved by discussing with the other two review authors (AU1 and AU4). We contacted original authors of studies to obtain clarifications when further information was required.

\section{Risk of Bias}

Two review authors (AU2 and AU3) independently assessed the risk of bias in the included studies. Methodological quality of RCTs was assessed using the Delphi list, a 9-item criteria list generated from the initial pool of 206 items using the Delphi consensus technique (Verhagen et al., 1998). The Delphi list mainly covers five domains including population, treatment allocation, blinding, prognostic comparability, and analysis. Quality of single-group pre-post studies and quasiexperimental study was assessed using the Risk of Bias Assessment Tool for Nonrandomized Studies (RoBANS), which contains six domains: selection of participants, confounding variables, measurement of exposure, blinding of outcome assessments, addressing incomplete outcome data, and selective outcome reporting (Kim et al., 2013). Two assessors (AU2 and AU3) rated each study as low, high, or unclear risk of bias for each of the domains, demonstrating an accepted level of inter-rater agreement (Cohen's kappa $=0.79)$. Any disagreements were resolved by consensus with the other two review authors (AU1 and AU4).

\section{Synthesis of Results and Meta-analyses}

All calculations were performed using Comprehensive Meta-Analysis (CMA) software. Separate meta-analyses were performed to calculate three effect sizes (Cohen's $d$ ) to indicate the effects of MBPG interventions on three outcome domains: family functioning, parental mental health, and child mental health. Small, medium, and large effect sizes are denoted by Cohen's $d$ values of $0.2,0.5$, and 0.8 , respectively (Cohen, 1992). Effect sizes were classified as minor when Cohen's $d$ values are smaller than 0.2 and larger than 0 . A study might be included in more than one meta-analysis 
if it contained more than one outcome domain. For each independent meta-analysis, effect sizes of relevant studies were combined. We computed an effect size as a standardized mean difference (SMD) between the means of an MBPG intervention group and a control group at posttest for controlled studies, or between the means before and after an MBPG intervention for single-group studies. To avoid including more than one effect size per construct per sample, we averaged the effect sizes within a study when one outcome domain was measured by multiple tests (Borenstein et al., 2009; Lipsey \& Wilson, 2001). We used a random effect model to pool effect sizes given the variations across MBPG interventions. A combined effect size was considered significant if the $p$ value was significant in the $z$ test and the confidence interval (CI) did not include zero. Precision of effect sizes was addressed by $95 \%$ CIs.

\section{Additional Analyses}

Heterogeneity across studies was tested using the $Q$ statistic and quantified by the I-squared $\left(I^{2}\right)$ value (Borenstein et al., 2009). Sensitivity analyses were conducted by removing studies one-by-one to estimate the effects of MBPG interventions on each outcome domain. We tested possible publication bias using the visual inspection of funnel plot asymmetry (Borenstein et al., 2009) and the Egger test (Sterne et al., 2001). Additionally, by using mixed effect models, moderator analyses were performed to explain the variability in effects of MBPG interventions across studies if the assumption of homogeneity between studies was rejected. Studies were grouped by relevant characteristics of participants, interventions, and studies to explore potential confounders.

\section{Results}

\section{Study Selection}

The study flow diagram is displayed in Fig. 1. Electronic database search yielded a total of 4,281 citations, of which 3,687 came from the initial search and 594 came from the second-round search. Twenty-eight additional records were identified through other sources, of which 26 came from hand-searching of the bibliographies of included studies and two came from consulting experts in the field of mindfulness. After de-duplication, 1,962 articles remained for title, abstract, and full-text screening, after which 19 articles remained. In one study, an MBPG intervention group was compared with both an active control group and a wait-list control group (Hancock et al., 2018). Since we were interested in the effects of MBPG interventions when comparing with both conditions, we treated this article as two separated studies. We labeled the MBPG-active control pair as study 1 and the MBPG-wait-list control pair as study 2. As a result, the current systematic review included 20 independent studies.

\section{Study Characteristics}

As shown in Table 1 , studies $(k=20)$ included in this systematic review targeted different types of youth and their parents. In terms of the characteristics of participating youth, 10 studies targeted adolescents (mean age $=12-17$ years), five studies targeted school-aged children (mean age $=6-11$ years), and one study targeted preschool children only (mean age $=3-5$ years; Jackman et al., 2019). Four studies were conducted among mixed age group combining children and adolescents. Boys had slightly more representation than girls; of the 18 studies in which researchers reported child gender, girls ranged from 17 to $92 \%$ (mean $\%=41.6 \%$ ). Of the seven studies that reported youth race/ ethnicity, members of ethnic minorities ranged 9-50\%, and there were more White youth than members of ethnic minorities (mean $\%$ of racial/ethnic minorities $=23.9 \%$ ). Most studies $(k=18)$ targeted youth with health issues. Specifically, 11 studies involved youth with developmental disorders such as ADHD and autism spectrum disorders (ASD), four studies involved youth with internalizing disorders such as depression and anxiety, one study involved youth with externalizing disorders such as oppositional defiant disorder (ODD) and conduct disorder (CD), and four studies targeted youth with physical health issues such as obesity and neurofibromatosis type 1 (NF1).

With regard to the characteristics of participating parents, only six studies provided their age information (mean age $=44.9$ years). Mothers were the most common parent participants in the studies. Among the 13 studies that reported parent gender, mothers accounted for $50 \%$ of participants or more in every study (mean $\%=72 \%$ ). Three studies involved parents who reported a history of mental health issues, such as ADHD, ASD, depression, and post-traumatic stress disorder (PTSD).

Participants of included studies were recruited from diverse settings, including clinical settings $(k=7)$ such as mental health centers, community and school settings $(k=6)$ such as integrated family service centers and elementary or middle schools, and others $(k=4)$ such as referrals from school counsellors and health professionals or recommendations by other parents. Of the six studies in which researchers reported residential areas, three targeted urban families and three involved both urban and rural families. Of the five studies that reported family socioeconomic status (SES), two studies targeted low-income population (Jackman et al., 2019 for Head Start families in the USA; Lo et al., 2019 for low-income families in Hong Kong). Besides one 


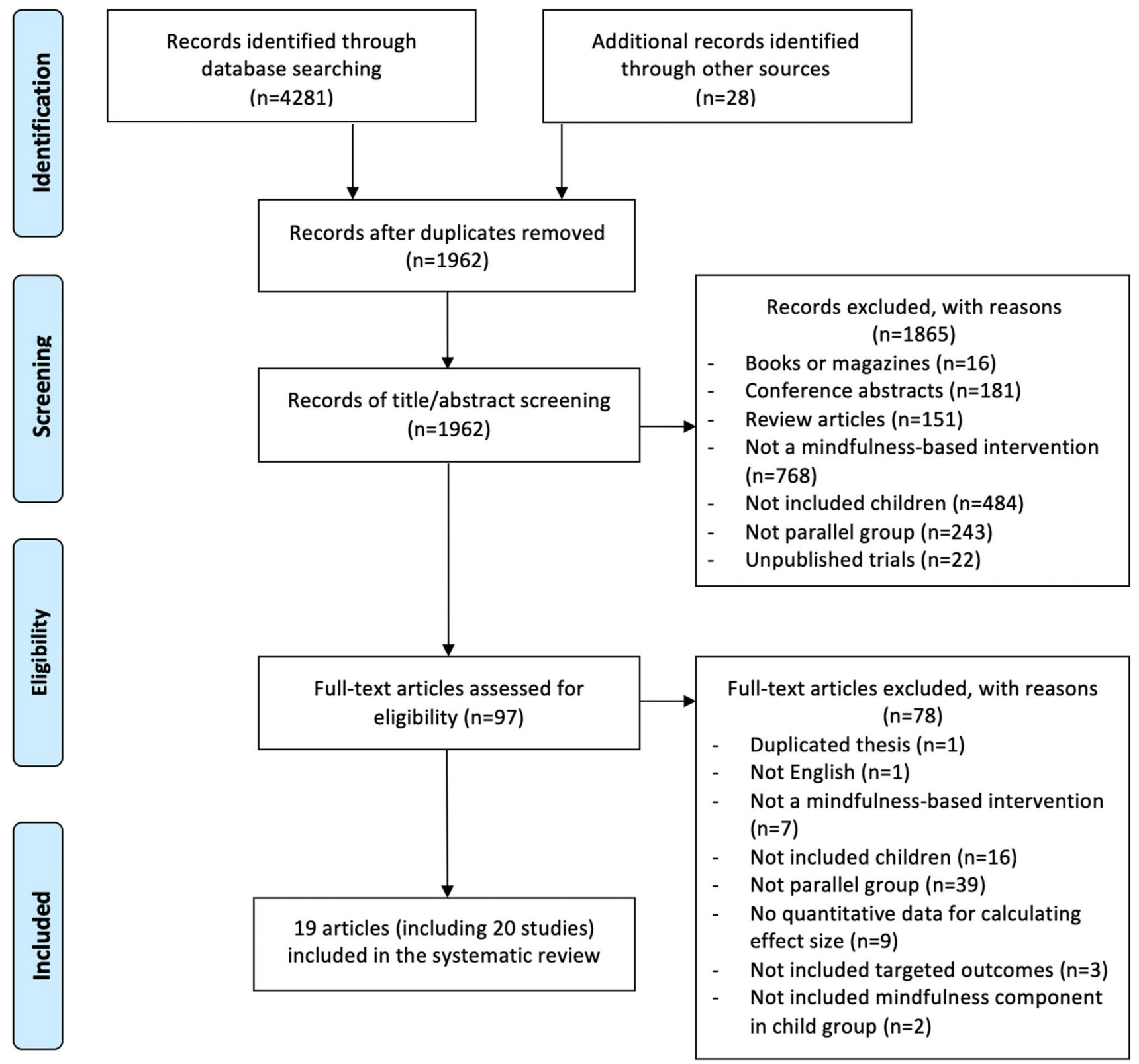

Fig. 1 Screening process of resources

study conducted in Mexico (López-Alarcón et al., 2020), the majority of families were recruited from developed countries or regions with very high Human Development Index (HDI) (indicated by an HDI of 0.8 or above; United Nations Development Programme, 2019), such as the USA $(k=5)$, the Netherlands $(k=5)$, Canada $(k=3)$, Hong Kong $(k=3)$, Australia $(k=2)$, and the UK $(k=1)$.

Although mindfulness was the main intervention component in both child and parent groups of all included studies, the specific intervention approaches were not exactly the same. In terms of child groups, intervention approaches such as mindfulness-based cognitive therapy (MBCT), mindfulness-based stress reduction (MBSR), acceptance and commitment therapy (ACT), and other manualized mindfulness programs were adapted. Two studies also involved other activities, such as conventional nutritional intervention (CNI; Jackman et al., 2019) and prosocial behavior learning activities based on the standard High Scope preschool curriculum (López-Alarcón et al., 2020). The dosage of child interventions varied from $6 \mathrm{~h}$ (e.g., Martin et al., 2016) to 16-24 h (Tronieri et al., 2019). Parent interventions could be classified into two major types: mindful parenting $(k=11)$ and other mindfulness $(k=9)$. In mindful parenting groups, mindfulness concepts and techniques were explicitly applied 


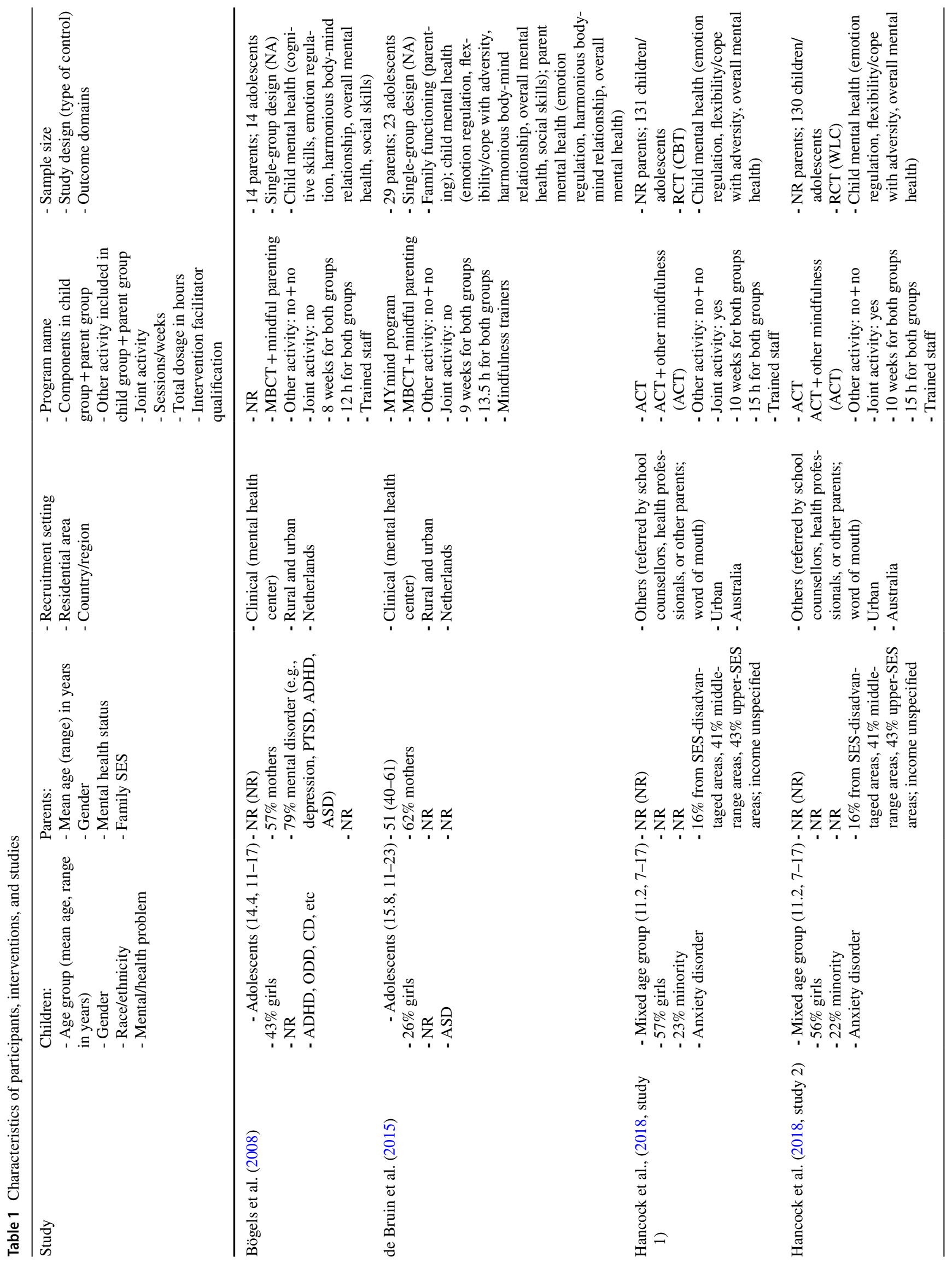




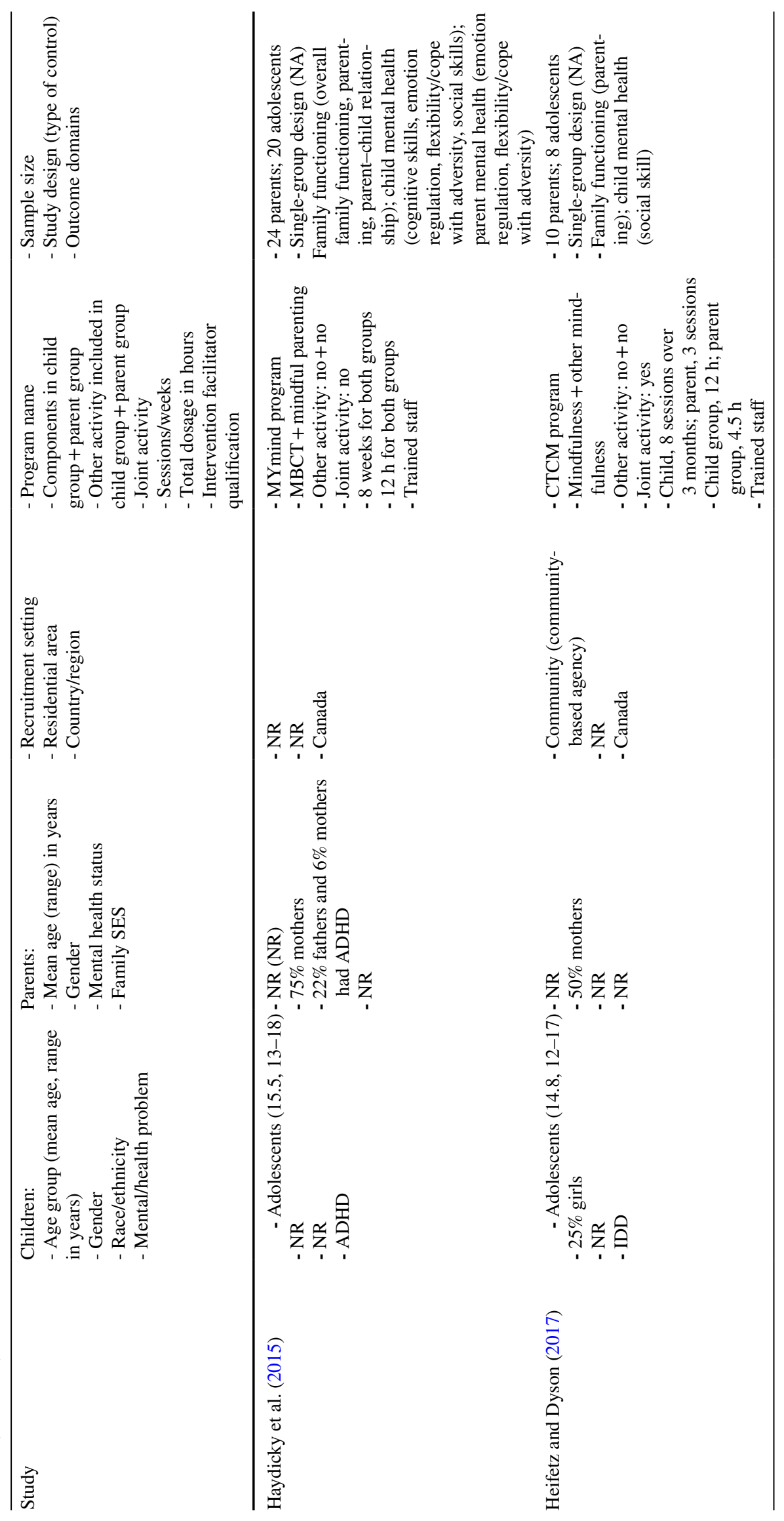




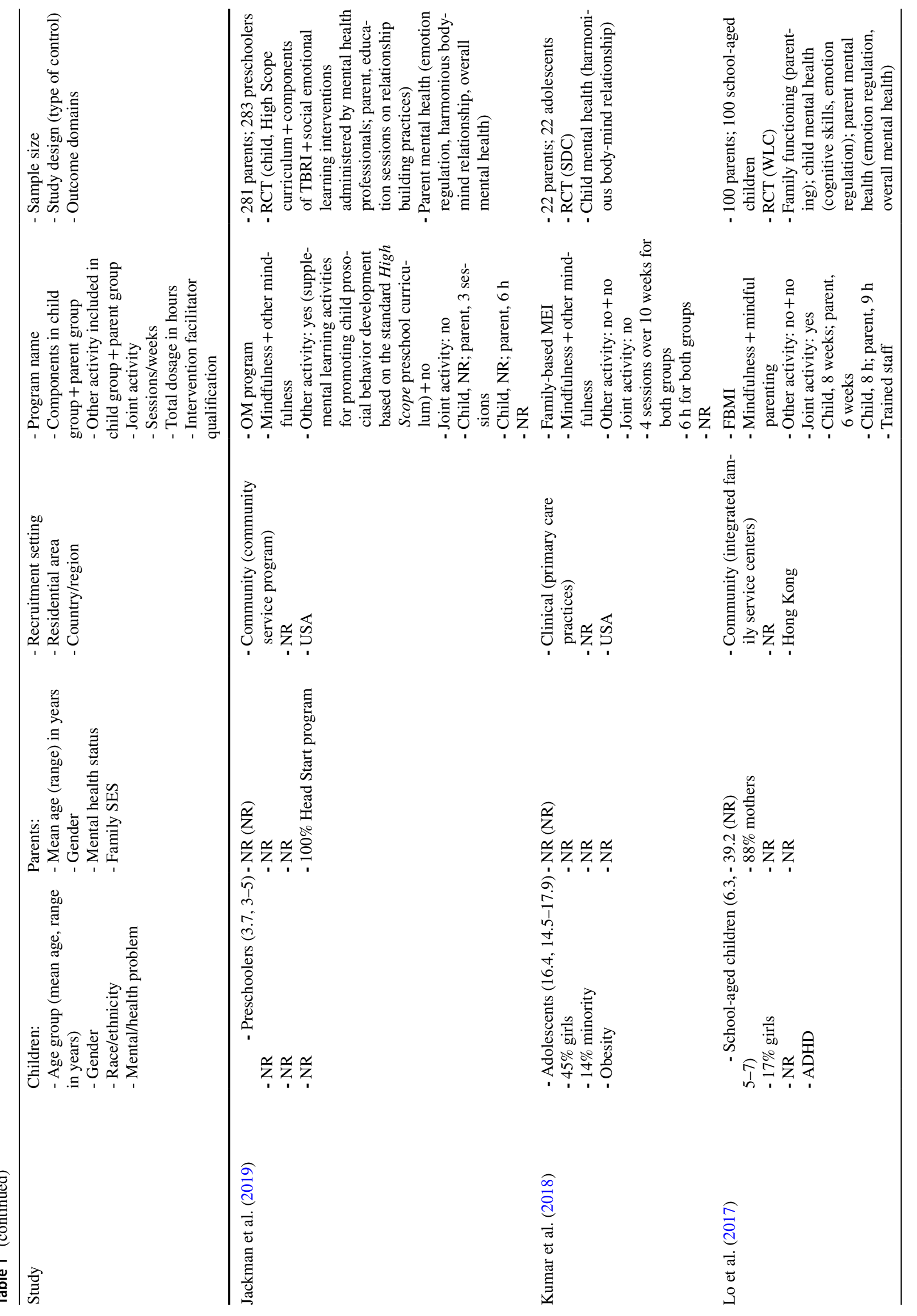




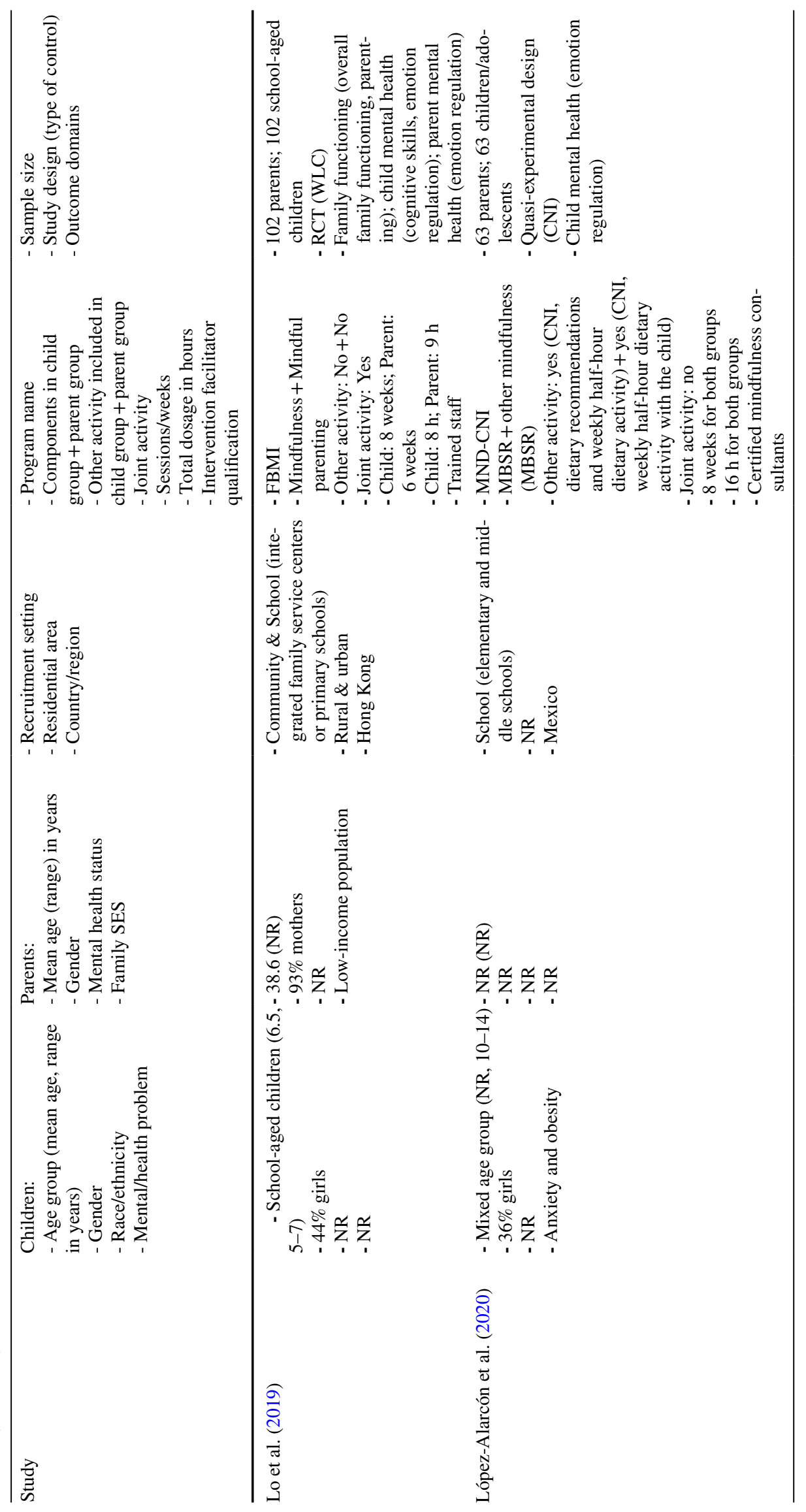




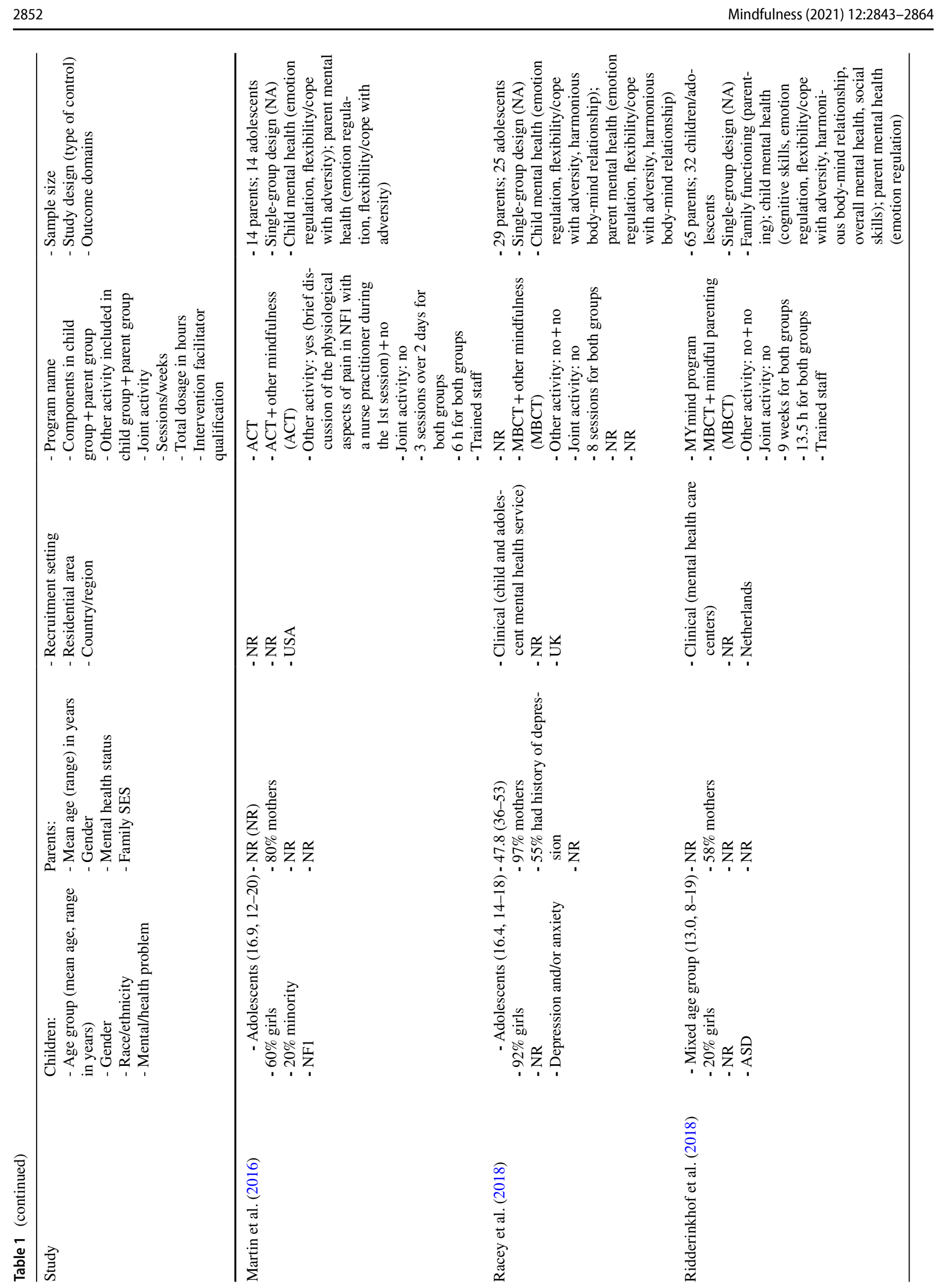

照 Springer 


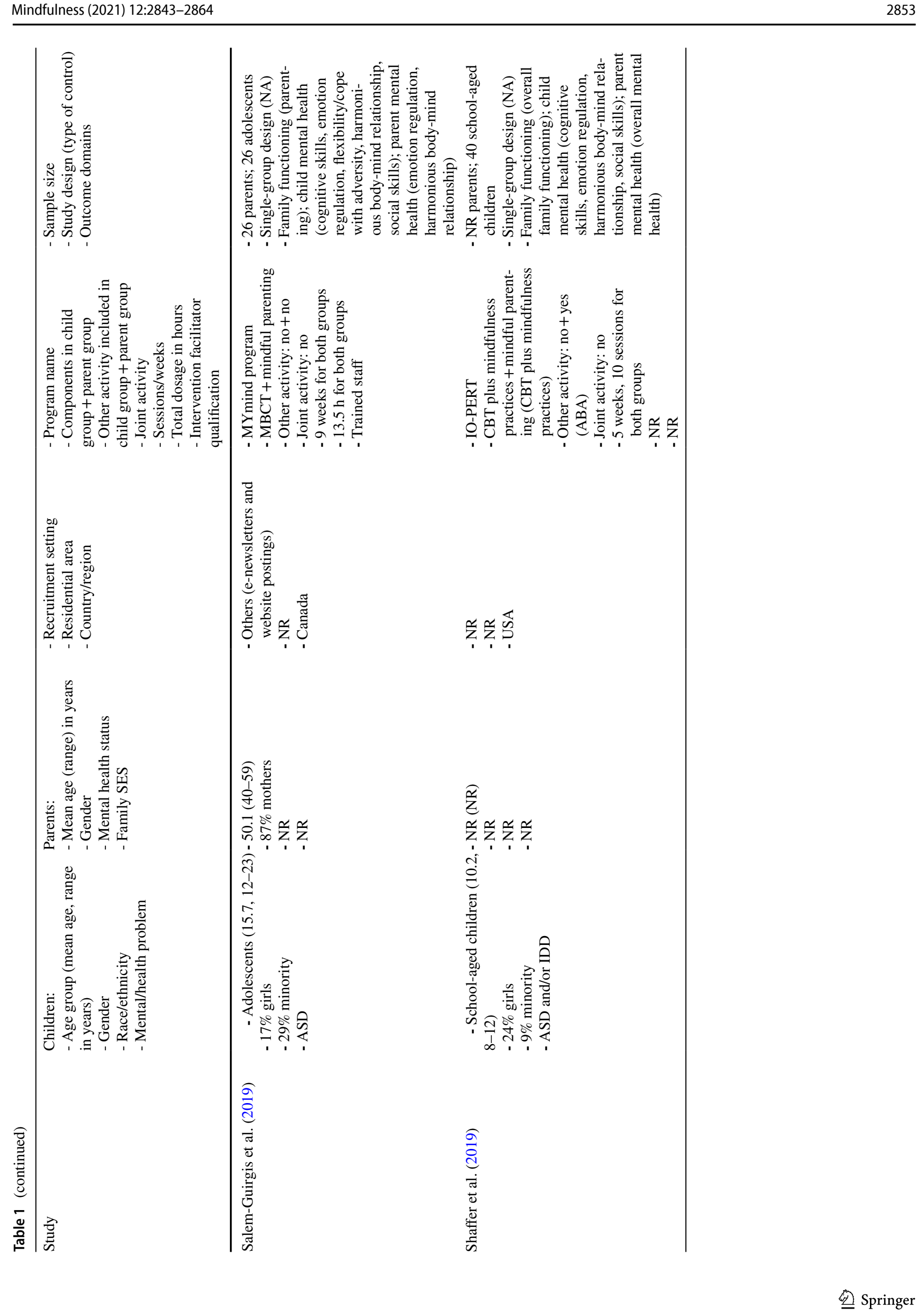




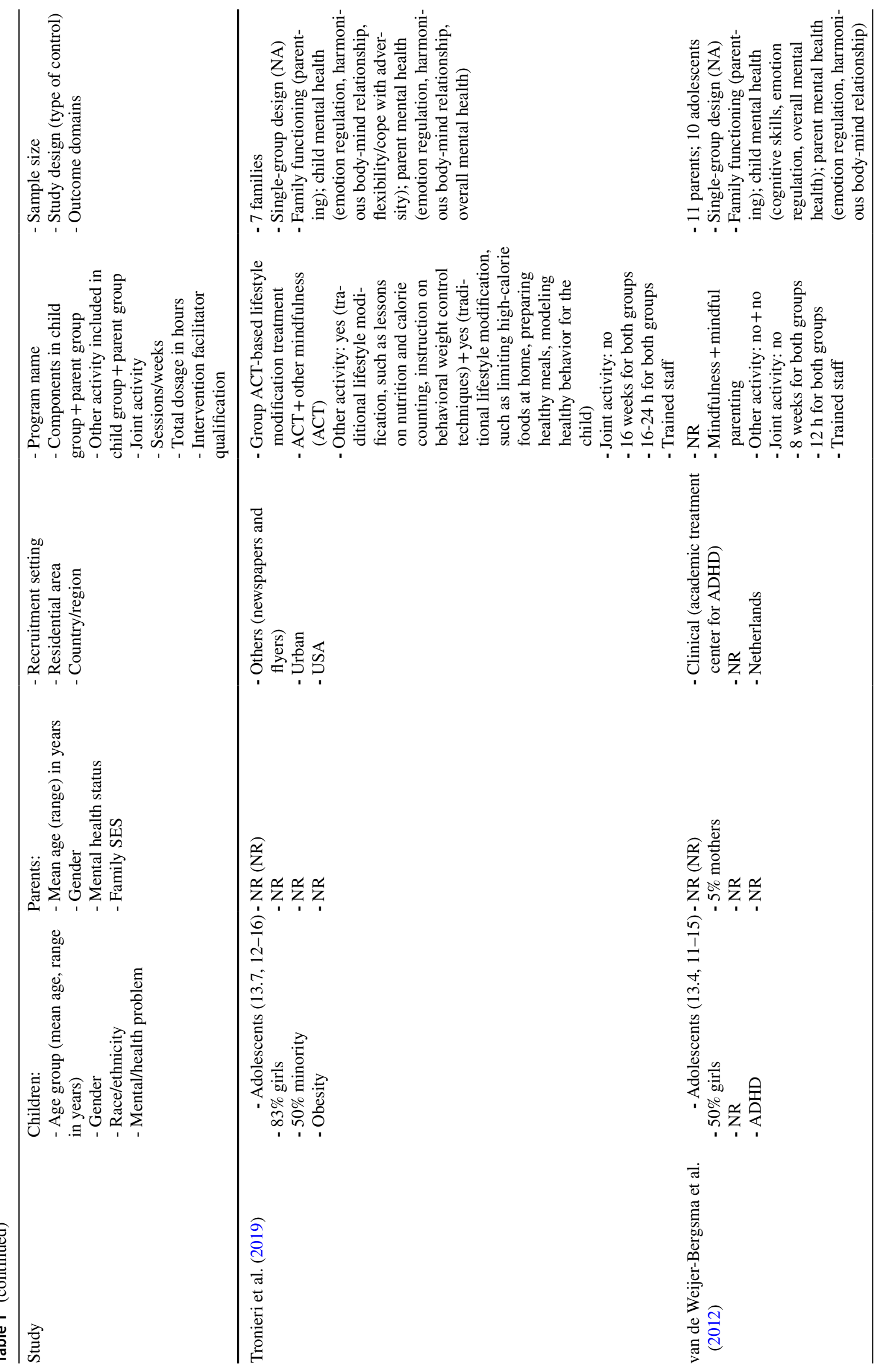




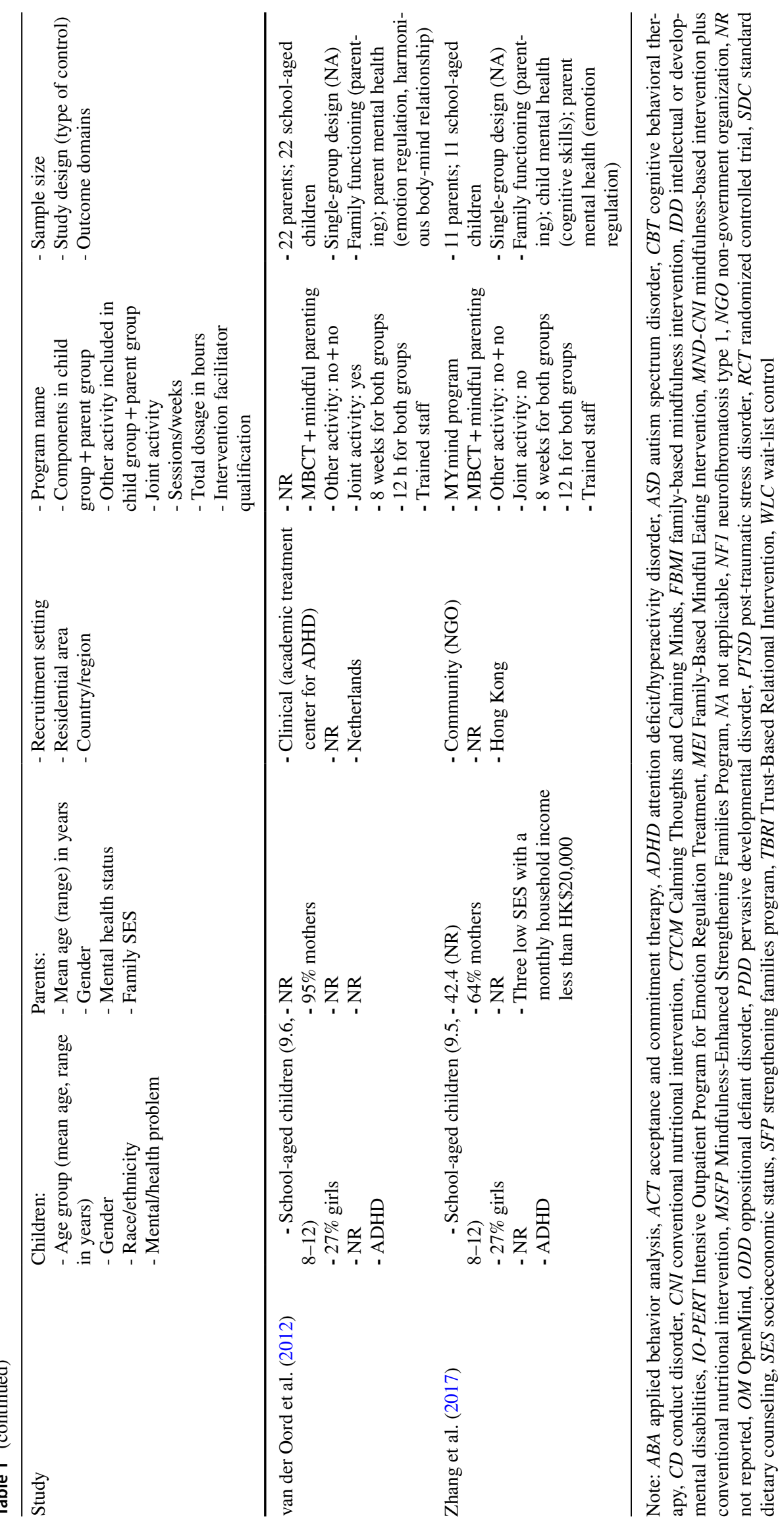


to parenting (e.g., mindful observation of the child, mindful parent-child communication); other activities such as applied behavior analysis (ABA; Shaffer et al., 2019) and traditional lifestyle modification (Tronieri et al., 2019) were also used in mindful parenting groups. In other mindfulness groups, mindfulness was not applied to parenting practices though it may have been utilized as a main component (e.g., for stress reduction). The dosage of parent interventions varied from $4.5 \mathrm{~h}$ (Heifetz \& Dyson, 2017) to 16-24 h (Tronieri et al., 2019). Although all interventions were delivered in parallel formats, some studies $(k=6)$ involved joint activities with children and their parents. Of 16 studies that reported qualification of staff, all interventions were delivered by instructors receiving mindfulness training, among which two interventions (de Bruin et al., 2015; López-Alarcón et al., 2020) were delivered by experienced experts (e.g., mindfulness trainers and certified mindfulness consultants).

The majority $(k=19)$ of included studies were published in or after 2010. Sample sizes ranged from seven (Tronieri et al., 2019) to 281 (Jackman et al., 2019) families, with the majority $(k=14)$ below 50 families; nearly one-third $(k=6)$ of the studies have a sample size of below 20 families. In terms of study design, there were 13 non-controlled studies (i.e., single-group pre-post studies) and seven controlled studies (six RCTs and one quasi-experimental study).

\section{Risk of Bias}

Summaries of risk of bias are presented in Supplementary Appendix I. The controlled studies overall showed low risk of bias in most domains, along with a few domains with unclear risk (i.e., without sufficient information to classify as high or low risk). Notably, the domains "was the care provider (intervention facilitator) blinded" and "was the patient (participant) blinded" contained the most high/unclear risk. The single-group pre-post studies showed relatively more domains with high or unclear risk, particularly for the "confounding variables," "measurement of exposure," and "blinding of outcome assessment" domains.

\section{Meta-analyses}

Figure 2 presents results of three separate meta-analyses on the pooled effects of MBPG interventions on family functioning, parental mental health, and child mental health. Table 2 displays outcome measures and effectiveness of each outcome domain. Detailed outcome measures are listed in Appendix II.

The meta-analysis combining results from 11 studies indicated a minor but significant post-intervention improvement in family functioning ( $d=0.182,95 \%$ CI $[0.045,0.319])$. The $z$-test result showed that the overall effect size differed significantly from zero $(z=2.600, p=0.009)$. The assumption of homogeneity between studies was not rejected as the $Q$ statistic of $16.496(p=0.086)$ was not statistically significant. Specifically, MBPG interventions demonstrated a significant effect in improving parenting behaviors $(d=0.227,95 \%$ CI $[0.179,0.275])$. Yet, a significant effect was not found on family functioning and relations $(d=0.207$, 95\% CI [ $-0.035,0.453])$ as the CI included zero.

Fourteen studies assessed the effects of MBPG interventions on parental mental health. The meta-analysis indicated a significant improvement in parental mental health with a weighted mean effect $d=0.238$ (95\% CI [0.110, 0.365]). The $z$-test results showed that the overall effect size differed significantly from zero $(z=3.656, p=0.000)$. The assumption of homogeneity between studies was not rejected $(Q=21.767, p=0.059)$. MBPG interventions showed small-to-medium effects on the improvement of parents' emotion regulation $(d=0.208)$, harmonious body-mind relationship $(d=0.570)$, and overall mental health $(d=0.332)$. Yet, MBPG interventions did not show a significant effect in improving parents' flexibility and ability to cope with adversity $(d=0.357,95 \%$ CI $[-0.004,0.718])$.

Combining results from 18 studies yielded a weighted mean effect on child mental health of $d=0.325$ (95\% CI $[0.137,0.513])$. The $z$-test result showed that the overall effect size differed significantly from zero $(z=3.387$, $p=0.001$ ). Thus, the MBPG interventions included in this meta-analysis had a statistically significant effect on child mental health. The statistically significant $Q$ statistic of $60.203(p=0.000)$ indicated that the differences among the effect sizes were due to heterogeneity rather than participant-level sampling error. The high $I^{2}$ value $\left(I^{2}=71.762\right)$ indicated that approximately $72 \%$ of total variance among studies was due to heterogeneity. Regarding specific outcome measures, six studies evaluated the effects of MBPG interventions on overall child mental health, showing a significant and medium combined effect $(d=0.528)$. MBPG interventions also showed small or minor positive effects on children's cognitive skills $(d=0.239)$, social skills $(d=0.044)$, and flexibility and ability to cope with adversity $(d=0.295)$. Yet, MBPG interventions showed a negative effect on the harmonious relationship between body and mind among children $(d=-0.165)$.

\section{Moderator Analyses}

Since the assumption of homogeneity between studies on child mental health was rejected, moderator analyses were undertaken to assess whether the characteristics of the participants, interventions, and studies could account for the variance in the effects of MBPG interventions. Results of univariate analysis of moderator variables for child mental health are presented in Table 3. Seven moderator variables might significantly contribute to between-group variance, 


\begin{tabular}{|c|c|c|c|c|c|c|c|}
\hline \multicolumn{8}{|c|}{ (A) Family Functioning $(k=11)$} \\
\hline \multirow[t]{2}{*}{$\underline{\text { Study name }}$} & \multicolumn{7}{|c|}{ Statistics for each study } \\
\hline & $\begin{array}{l}\text { Std diff } \\
\text { in means }\end{array}$ & $\begin{array}{l}\text { Standard } \\
\text { error }\end{array}$ & Variance & $\begin{array}{l}\text { Lower } \\
\text { limit }\end{array}$ & $\begin{array}{l}\text { Upper } \\
\text { limit }\end{array}$ & z-Value & $\mathrm{p}$-Value \\
\hline de Bruin et al., 2015 & 0.225 & 0.026 & 0.001 & 0.174 & 0.276 & 8.689 & 0.000 \\
\hline Haydicky et al., 2015 & 0.139 & 0.301 & 0.091 & -0.452 & 0.729 & 0.460 & 0.646 \\
\hline Heifetz et al., 2 & 1.278 & 1.171 & 1.372 & -1.018 & 3.574 & 1.091 & 0.275 \\
\hline Lo et al., 2019 & 0.446 & 0.200 & 0.040 & 0.053 & 0.839 & 2.224 & 0.026 \\
\hline Ridderinkhof et al., 2018 & 0.485 & 0.115 & 0.013 & 0.259 & 0.711 & 4.213 & 0.000 \\
\hline Salem-Guirgis et al., 2019 & 0.040 & 0.150 & 0.023 & -0.254 & 0.334 & 0.267 & 0.790 \\
\hline Shaffer et al. 2019 & 0.042 & 0.186 & 0.035 & -0.323 & 0.406 & 0.224 & 0.823 \\
\hline Tronieri et al., 2019 & 0.037 & 0.576 & 0.332 & -1.092 & 1.165 & 0.064 & 0.949 \\
\hline van de Weijer-Bergsma et al., 2012 & -0.252 & 0.397 & 0.158 & -1.030 & 0.526 & -0.635 & 0.525 \\
\hline van der Oord et al., 2012 & -0.221 & 0.216 & 0.047 & -0.645 & 0.203 & -1.020 & 0.308 \\
\hline Zhang et al., 2017 & -0.083 & 0.307 & 0.094 & -0.685 & 0.518 & -0.272 & 0.786 \\
\hline Random effect & 0.182 & 0.070 & 0.005 & 0.045 & 0.319 & 2.600 & 0.009 \\
\hline
\end{tabular}

\section{(B) Parental Mental Health $(k=14)$}

\begin{tabular}{|c|c|c|c|c|c|c|c|}
\hline \multirow[t]{2}{*}{$\underline{\text { Study name }}$} & \multicolumn{7}{|c|}{ Statistics for each study } \\
\hline & $\begin{array}{l}\text { Std diff } \\
\text { in means }\end{array}$ & $\begin{array}{l}\text { Standard } \\
\text { error }\end{array}$ & Variance & $\begin{array}{c}\text { Lower } \\
\text { limit }\end{array}$ & $\begin{array}{c}\text { Upper } \\
\text { limit }\end{array}$ & Z-Value & $\mathrm{p}$-Value \\
\hline de Bruin et al., 2015 & 0.377 & 0.026 & 0.001 & 0.327 & 0.427 & 14.729 & 0.000 \\
\hline Haydicky et al., 2015 & 0.141 & 0.341 & 0.116 & -0.527 & 0.809 & 0.414 & 0.679 \\
\hline Jackman et al., 2019 & -0.143 & 0.332 & 0.110 & -0.794 & 0.507 & -0.432 & 0.666 \\
\hline Lo et al., 2017 & 0.131 & 0.200 & 0.040 & -0.261 & 0.524 & 0.656 & 0.512 \\
\hline Lo et al., 2019 & 0.328 & 0.200 & 0.040 & -0.063 & 0.720 & 1.646 & 0.100 \\
\hline Martin et al., 2016 & -0.045 & 0.451 & 0.203 & -0.929 & 0.838 & -0.100 & 0.920 \\
\hline Racey et al., 2018 & 0.577 & 0.280 & 0.078 & 0.028 & 1.125 & 2.062 & 0.039 \\
\hline Ridderinkhof et al., 2018 & 0.260 & 0.100 & 0.010 & 0.064 & 0.456 & 2.600 & 0.009 \\
\hline Salem-Guirgis et al., 2019 & 0.250 & 0.160 & 0.026 & -0.064 & 0.564 & 1.563 & 0.118 \\
\hline Shaffer et al. 2019 & 0.114 & 0.186 & 0.035 & -0.251 & 0.480 & 0.614 & 0.539 \\
\hline Tronieri et al., 2019 & 0.298 & 0.576 & 0.331 & -0.830 & 1.426 & 0.518 & 0.604 \\
\hline van de Weijer-Bergsma et al., 2012 & 0.261 & 0.370 & 0.137 & -0.463 & 0.986 & 0.707 & 0.480 \\
\hline van der Oord et al., 2012 & 0.408 & 0.222 & 0.049 & -0.027 & 0.843 & 1.839 & 0.066 \\
\hline Zhang et al., 2017 & -0.926 & 0.360 & 0.130 & -1.632 & -0.219 & -2.569 & 0.010 \\
\hline Random effect & 0.238 & 0.065 & 0.004 & 0.110 & 0.365 & 3.656 & 0.000 \\
\hline
\end{tabular}
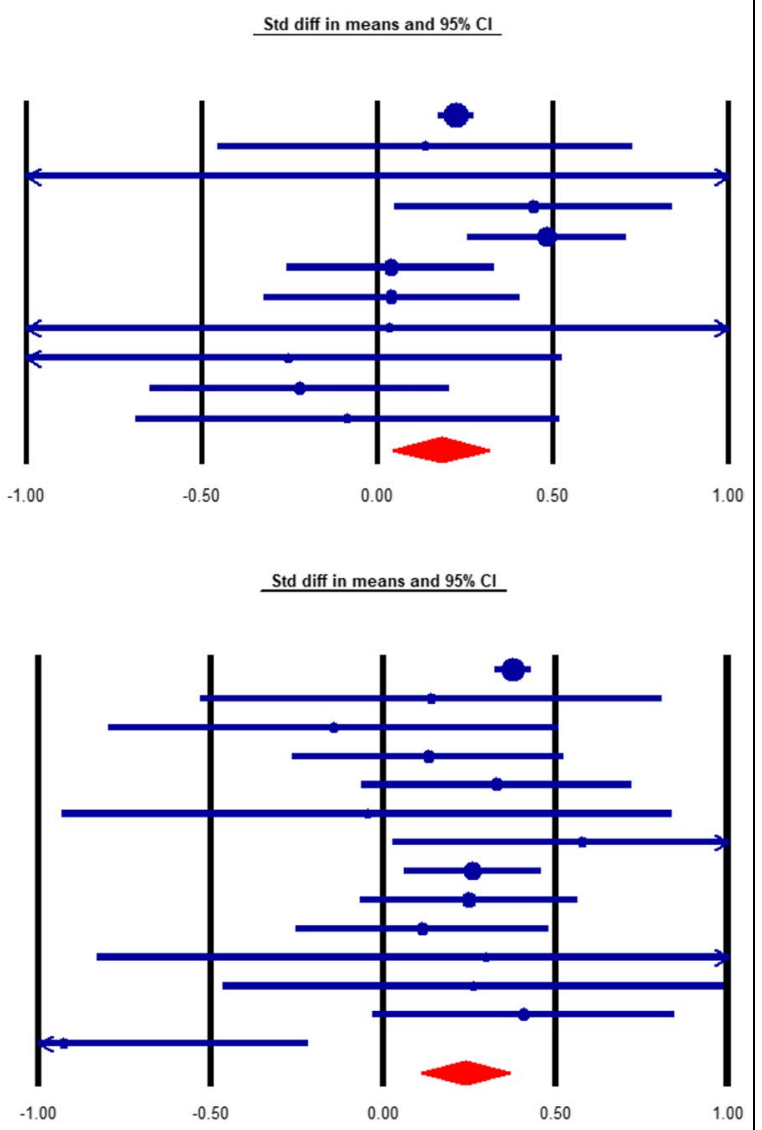

\section{(C) Child Mental Health $(k=18)$}

Study name
Bogels et al., 2008
de Bruin et al., 2015
Hancock et al., 2018 (study 1)
Hancock et al., 2018 (study 2)
Haydicky et al., 2015
Heifetz et al., 2017
Kumar et al., 2018
Lo et al., 2017
Lo et al., 2019
Lopez-Alarcon et al., 2020
Martin et al., 2016
Racey et al., 2018
Ridderinkhof et al., 2018
Salem-Guirgis et al., 2019
Shaffer et al. 2019
Tronieri et al., 2019
van de Weijer-Bergsma et al., 2012
Zhang et al., 2017
Random effect

\begin{tabular}{rcrccrr} 
& & \multicolumn{5}{c}{ Statistics for each study } \\
\cline { 3 - 7 } Std diff & Standard & & Lower & Upper & \\
in means & error & Variance & limit & limit & Z-Value & p-Value \\
0.329 & 0.343 & 0.117 & -0.343 & 1.000 & 0.960 & 0.337 \\
0.090 & 0.035 & 0.001 & 0.021 & 0.159 & 2.560 & 0.010 \\
-0.104 & 0.176 & 0.031 & -0.448 & 0.241 & -0.589 & 0.556 \\
0.809 & 0.183 & 0.033 & 0.450 & 1.167 & 4.419 & 0.000 \\
0.283 & 0.300 & 0.090 & -0.304 & 0.870 & 0.944 & 0.345 \\
-0.312 & 0.696 & 0.484 & -1.675 & 1.051 & -0.449 & 0.654 \\
-0.368 & 0.443 & 0.197 & -1.237 & 0.501 & -0.829 & 0.407 \\
0.153 & 0.200 & 0.040 & -0.239 & 0.546 & 0.765 & 0.444 \\
0.141 & 0.198 & 0.039 & -0.248 & 0.529 & 0.709 & 0.478 \\
2.667 & 0.439 & 0.193 & 1.806 & 3.527 & 6.076 & 0.000 \\
0.253 & 0.437 & 0.191 & -0.604 & 1.110 & 0.579 & 0.562 \\
0.851 & 0.327 & 0.107 & 0.209 & 1.492 & 2.599 & 0.009 \\
0.261 & 0.181 & 0.033 & -0.093 & 0.616 & 1.443 & 0.149 \\
0.164 & 0.140 & 0.020 & -0.111 & 0.439 & 1.172 & 0.241 \\
0.385 & 0.187 & 0.035 & 0.018 & 0.752 & 2.058 & 0.040 \\
0.082 & 0.668 & 0.446 & -1.227 & 1.391 & 0.123 & 0.902 \\
0.342 & 0.351 & 0.123 & -0.347 & 1.030 & 0.973 & 0.330 \\
0.310 & 0.353 & 0.125 & -0.382 & 1.002 & 0.878 & 0.380 \\
0.325 & 0.096 & 0.009 & 0.137 & 0.513 & 3.387 & 0.001
\end{tabular}

Fig. 2 Effects of mindfulness-based parallel-group interventions on family functioning (A), parental mental health (B), and child mental health (C)

which were child age (adolescents $[d=0.106]<$ schoolaged children $[d=0.240]<$ mixed age group $[d=0.435]$; $Q_{b}=10.400, p<0.01$ ), child gender (predominantly male $[d=0.124]<$ predominantly female $[d=0.379] ; Q_{b}=5.195$, $p<0.05$ ), recruitment setting (clinical $[d=0.106]<$ community $[d=0.153]<$ others $[d=0.255]<\operatorname{school}[d=2.667]$; 
Table 2 Outcome measures and effectiveness

\begin{tabular}{|c|c|c|c|c|c|c|}
\hline \multirow[t]{2}{*}{ Outcomes } & \multirow[t]{2}{*}{$k$} & \multirow{2}{*}{$\begin{array}{l}\text { Effect size } \\
\text { Cohen's } d(95 \% \mathrm{CI})\end{array}$} & \multicolumn{4}{|c|}{ Heterogeneity } \\
\hline & & & $Q$ & $d f$ & $p$ & $I^{2}$ \\
\hline Family functioning & 11 & $0.182(0.045,0.319)$ & 16.496 & 10 & 0.086 & 39.380 \\
\hline Parenting behaviors & 8 & $0.227(0.179,0.275)$ & 18.047 & 7 & 0.012 & 61.213 \\
\hline Family functioning or relations & 3 & $0.207(-0.035,0.453)$ & 2.881 & 2 & 0.237 & 30.569 \\
\hline Parental mental health & 14 & $0.238(0.110,0.365)$ & 21.767 & 13 & 0.059 & 40.276 \\
\hline Emotion regulation & 13 & $0.208(0.180,0.237)$ & 23.585 & 12 & 0.023 & 49.120 \\
\hline Flexibility and ability to cope with adversity & 3 & $0.357(-0.004,0.718)$ & 1.911 & 2 & 0.385 & 0.000 \\
\hline Harmonious relationship between body and mind & 7 & $0.570(0.530,0.611)$ & 10.791 & 6 & 0.095 & 44.399 \\
\hline Overall mental health & 5 & $0.332(0.264,0.400)$ & 1.445 & 4 & 0.836 & 0.000 \\
\hline Child mental health & 18 & $0.325(0.137,0.513)$ & 60.203 & 17 & 0.000 & 71.762 \\
\hline Cognitive skills & 8 & $0.239(0.115,0.363)$ & 4.342 & 7 & 0.740 & 0.000 \\
\hline Social skills & 7 & $0.044(0.003,0.085)$ & 26.949 & 6 & 0.000 & 77.735 \\
\hline Emotion regulation & 15 & $0.066(-0.001,0.133)$ & 76.926 & 14 & 0.000 & 81.801 \\
\hline Flexibility and ability to cope with adversity & 9 & $0.295(0.219,0.371)$ & 33.005 & 8 & 0.000 & 75.761 \\
\hline Harmonious relationship between body and mind & 9 & $-0.165(-0.244,-0.094)$ & 30.558 & 8 & 0.000 & 73.820 \\
\hline Overall mental health & 6 & $0.528(0.459,0.597)$ & 20.655 & 5 & 0.001 & 75.792 \\
\hline
\end{tabular}

Note: $95 \% \mathrm{CI}=$ lower and upper limits if $95 \%$ confidence interval

$Q_{b}=35.653, p<0.001$ ), type of parent group (mindful parenting group $[d=0.118]<$ other mindfulness group $\left.[d=0.455] ; Q_{b}=9.508, p<0.01\right)$, other activities in child group (no $[d=0.134]<$ yes $[d=1.212]$; $Q_{b}=14.562, p<0.001$ ), other activities in parent group (no $[d=0.128]<$ yes $\left.[d=0.696] ; Q_{b}=11.231, p<0.01\right)$, and study design (non-controlled studies $[d=0.123]<$ controlled studies $\left.[d=0.327] ; Q_{b}=4.520, p<0.05\right)$. Other variables including residential area (urban area vs. mixed area) and joint parent-child activity (yes vs. no) might not significantly predict the effects of MBPG interventions on child mental health.

\section{Publication Bias}

Figure 3 presents the visual illustration of the funnel plots. The assessments of publication bias for three outcome domains showed obscure asymmetry. Regarding the effect on family functioning, the findings of the Egger test indicated no significant publication bias $(t=0.739, p=0.479)$. Most studies were distributed symmetrically around the combined effect size and appeared toward the top of the funnel graph. However, there was one small study that published a larger effect (Heifetz \& Dyson, 2017), which might make the calculated effect size larger than the unbiased estimate. For the effect on child mental health, the results of the Egger test showed no significant publication bias $(t=1.953$, $p=0.068$ ), while a few studies concentrated on the right side of the mean effect size in the funnel plot, which indicated that the calculated effect size might also be larger than the unbiased effect size. Significant publication bias might exist in the effect on parent mental health $(t=2.735, p=0.018)$. The majority of studies appeared toward the top of the funnel plot, while one study appeared toward the bottom of the graph. A few studies concentrated on the left side of the mean effect size, which might make the calculated effect size smaller than the unbiased effect size. In sum, there might be a gap between the real effectiveness and the calculated effectiveness due to publication bias.

\section{Discussion}

Parent and child are interdependent components within the family system. This systematic review empirically supports that MBPG interventions simultaneously involving parent and child may holistically benefit individual well-being while improving integrated family systems. By pooling data from 20 interventions and representing 1,083 children and 1,131 parents, the meta-analyses found that MBPG interventions showed minor-to-small, significant, and positive effects on family functioning $(d=0.182)$, parental mental health $(d=0.238)$, and child mental health $(d=0.325)$. It is important to note that MBPG interventions showed positive impact on overall family functioning, a finding that was not mentioned in parent- and child-only MBIs. Although these family improvements are small in effect size, they may promote a nurturing home environment that fosters continued changes in individual family members in the long term (Lo et al., 2019; Shaffer et al., 2019).

Indeed, mindfulness may benefit the entire family system conceptually. For adults and children as individual 
Table 3 Univariate analysis of moderator variables for child mental health $(k=18)$

\begin{tabular}{|c|c|c|c|c|c|c|}
\hline \multirow[t]{2}{*}{ Moderators } & \multirow[t]{2}{*}{$k$} & \multirow[t]{2}{*}{ Effect size $(95 \% \mathrm{CI})$} & \multicolumn{4}{|l|}{ Heterogeneity } \\
\hline & & & $Q_{w}$ & $d f$ & $p$ & $I^{2}$ \\
\hline Child age & 18 & & $Q_{b}=10.400 * *$ & & 0.006 & \\
\hline School-aged children (mean age $=6-11$ years) & 4 & $0.240(0.030,0.451)$ & 1.081 & 3 & 0.782 & 0.000 \\
\hline Adolescents (mean age $=12-17$ years) & 10 & $0.106(0.041,0.171)$ & 8.396 & 9 & 0.495 & 0.000 \\
\hline Mixed age group & 4 & $0.435(0.237,0.633)$ & 40.326 & 3 & 0.000 & 92.561 \\
\hline Child gender & 17 & & $Q_{b}=5.195^{*}$ & & $\mathbf{0 . 0 2 3}$ & \\
\hline Predominantly male (female $<50 \%$ ) & 11 & $0.124(0.062,0.186)$ & 39.387 & 10 & 0.000 & 74.611 \\
\hline Predominantly female (female $\geq 50 \%$ ) & 6 & $0.379(0.169,0.589)$ & 15.411 & 5 & 0.009 & 67.555 \\
\hline Recruitment setting & 15 & & $Q_{b}=35.653^{* * *}$ & & 0.000 & \\
\hline Community & 4 & $0.153(-0.099,0.405)$ & 0.648 & 3 & 0.885 & 0.000 \\
\hline School & 1 & $2.667(1.806,3.527)$ & 0.000 & 0 & 1.000 & 0.000 \\
\hline Clinical & 6 & $0.106(0.040,0.173)$ & 8.134 & 5 & 0.149 & 38.531 \\
\hline Others & 4 & $0.255(0.072,0.437)$ & 13.793 & 3 & 0.003 & 78.251 \\
\hline Residential area & 6 & & $Q_{b}=3.212$ & & 0.073 & \\
\hline Urban area & 3 & $0.326(0.081,0.570)$ & 13.056 & 2 & 0.001 & 84.681 \\
\hline Mixed area & 3 & $0.094(0.026,0.161)$ & 0.528 & 2 & 0.764 & 0.000 \\
\hline Type of parent group & 18 & & $Q_{b}=9.508 * *$ & & 0.002 & \\
\hline Mindful parenting group & 10 & $0.118(0.056,0.180)$ & 4.835 & 9 & 0.848 & 0.000 \\
\hline Other mindfulness group & 8 & $0.455(0.250,0.660)$ & 45.860 & 7 & 0.000 & 84.736 \\
\hline Joint parent-child activity & 18 & & $Q_{b}=1.134$ & & 0.287 & \\
\hline Yes & 5 & $0.240(0.057,0.423)$ & 14.540 & 4 & 0.006 & 72.491 \\
\hline No & 13 & $0.135(0.073,0.198)$ & 44.529 & 12 & 0.000 & 73.051 \\
\hline Other activities in child group & 18 & & $Q_{b}=14.562 * * *$ & & $\mathbf{0 . 0 0 0}$ & \\
\hline Yes & 3 & $1.212(0.662,1.763)$ & 18.652 & 2 & 0.000 & 89.277 \\
\hline No & 15 & $0.134(0.074,0.193)$ & 26.989 & 14 & 0.019 & 48.126 \\
\hline Other activity in parent group & 18 & & $Q_{b}=11.231 * *$ & & 0.001 & \\
\hline Yes & 3 & $0.696(0.369,1.023)$ & 23.755 & 2 & 0.000 & 91.581 \\
\hline No & 15 & $0.128(0.067,0.188)$ & 25.2172 & 14 & 0.032 & 44.482 \\
\hline Study design & 18 & & $Q_{b}=4.520^{*}$ & & $\mathbf{0 . 0 3 3}$ & \\
\hline Controlled studies & 6 & $0.327(0.150,0.504)$ & 45.428 & 5 & 0.000 & 88.994 \\
\hline Non-controlled studies & 12 & $0.123(0.061,0.186)$ & 10.255 & 11 & 0.508 & 0.000 \\
\hline
\end{tabular}

Note: $95 \% \mathrm{CI}=$ lower and upper limits if $95 \%$ confidence interval; $Q_{w} / Q_{b}=$ test for homogeneity of effect sizes within (w) and between (b) groups; ${ }^{*} p<.05, * * p<.01, * * * p<.001$

members, mindfulness may embody as more awareness and acceptance of their thoughts, feelings, and actions without judgment, which is related to improved mental health status (Greco et al., 2011; Neece, 2014; Potharst et al., 2021). These improvements may directly and indirectly increase emotional support for other members within the family system, leading to a benign circle of mental well-being. Moreover, mindfulness could foster nonjudgmental acceptance of oneself and others, which may encourage greater emotional awareness, stronger compassion, more attentive listening, and flexibility in the parenting process (Duncan et al., 2015; Kabat-Zinn \& Kabat-Zinn, 1997). All of these could cultivate an open attitude in parent-child interactions and improve parent-child relationship quality (Duncan et al., 2015). This is in line with a previous study finding that both young people and their parents found the shared experience of attending MBPG intervention mutually supportive, which helped rebuild the impaired parent-child relationship (Racey et al., 2018).

The advantages of parallel-group format might play an important role in the effects of MBPG interventions. First, the parallel approach could create a shared understanding and sense of support between parents and children and improved the intergenerational aspects of depression (Racey et al., 2018). Also, the parallel approach could improve intervention adherence of participants. For instance, some hard-to-reach parents, such as parents with high ADHD levels, were less likely to respond to behavioral parent training but appeared to be more willing to participate in MBPG intervention for the purpose of improving their children's 
Fig. 3 Publication bias: funnel plot of standard error by standardized difference in means. Note: Publication bias was assessed by funnel plot for the effects of MBPG interventions on family functioning (A), parental mental health (B), and child mental health $(\mathbf{C})$. In a scatter plot, the unfilled circles represent included studies, the $\mathrm{x}$-axis represents the mean result (standardized difference in means), and the $y$-axis represents study precision (the standard errors of the effect estimates). Studies with large effect are placed at the right side and studies with small effect at the left side. The scale of the $y$-axis is reversed. Studies with low precision are placed at the bottom and studies with greater precision at the top of the plot

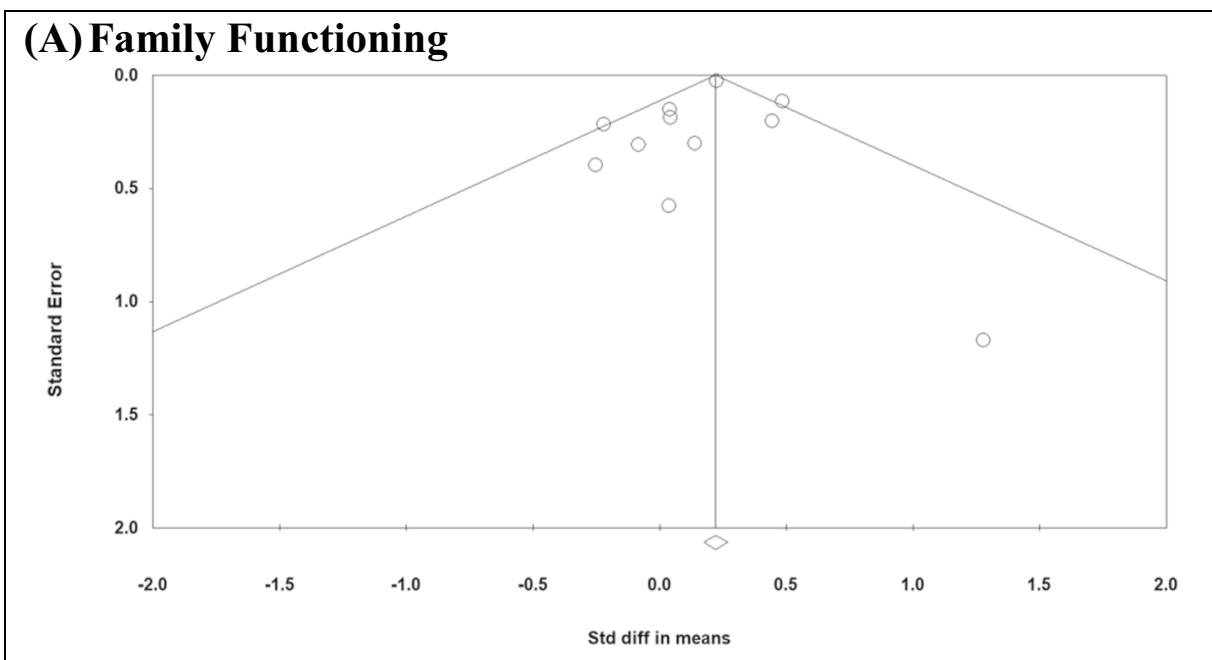

(B) Parental Mental Health

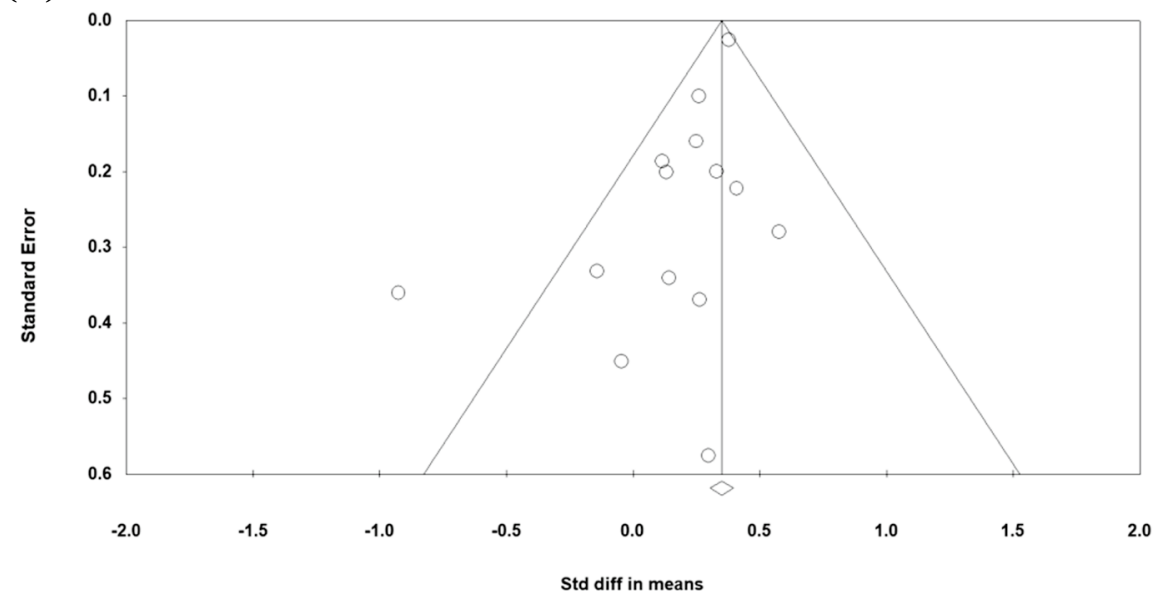

(C) Child Mental Health

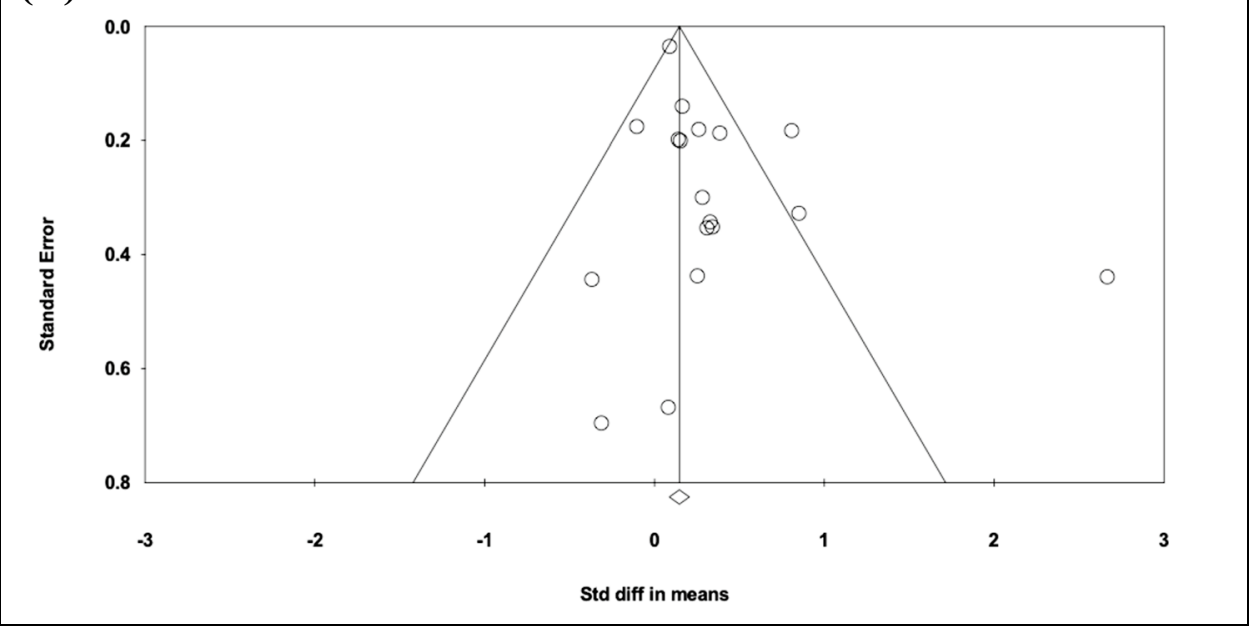

functioning rather than only targeting themselves (van der Oord et al., 2012). In a family-based mindful eating intervention, the attendance rate was $100 \%$ likely because involvement of the entire family was required (Kumar et al., 2018). In addition, family members could also reinforce each other's sustained practice, as parents could play a crucial role in modeling mindfulness techniques at home, thus maintaining regular child mindfulness practices and enhancing child treatment adherence (Haydicky et al., 2015; Heifetz \& Dyson, 2017; Martin et al., 2016). 
Despite these advantages, the effects found in our review are slightly smaller than previous meta-analyses of MBIs for parents on reducing parenting stress (Burgdorf et al., 2019) and MBIs delivered to youth in school settings (Zenner et al., 2014). In other words, the current meta-analysis does not support that MBPG interventions have strengthened effects for parents and children. One possible reason is that the smaller sample size and study design may have reduced statistical power in our significance tests. While the majority of our reviewed studies had below 50 families and used single-group designs, the majority of school-based MBIs in the previous review had over 50 participants and mostly used controlled designs (see Zenner et al., 2014).

Another possible explanation of the smaller effect size may be the involvement of clinical child samples. A recent meta-analysis showed that mindful parenting interventions had significant medium effect size on parenting mindfulness among parents with non-clinical child samples $(d=0.62)$, whereas no intervention effect was found for parents with clinical child samples $(d=0.05$; Shorey $\& \mathrm{Ng}, 2021)$. While $90 \%$ of studies ( $k=18$ out of 20 ) in our review involved clinical child samples (e.g., children with ADHD, ASD, depression), Zenner et al.'s (2014) review targeted a non-clinical setting (i.e., schools), and Burgdorf et al.'s (2019) review involved only three studies with clinical child samples (i.e., children with ADHD) among the 15 parent-only MBIs. It is possible that parents of children with developmental challenges may find it more difficult to achieve positive changes through MBPG interventions. While the small number of studies in our study did not allow for such subgroup comparison, future research may further examine this difference between clinical and non-clinical child samples.

With respect to the content and format of MBPG interventions, combining mindfulness components with other targeted activities in parent or child group might improve the effectiveness of MBPG interventions, especially for populations with health concerns, such as dietary recommendation or lifestyle modification for children with obesity (López-Alarcón et al., 2020; Tronieri et al., 2019), and discussions of pain-related physiology for adolescents with NF1 (Martin et al., 2016). However, our assumption that joint parent-child activities would make interventions more effective was rejected. Although interventions with joint activity did show greater effect on child mental health than interventions without joint activity ( $d=0.24$ vs. 0.135 ), this difference was not statistically significant. There are several possible explanations. First, some exercises for parents may need to be further modified for use among children and adolescents due to differences in cognitive and behavioral development. For instance, unlike with adults, most adolescents could not inhibit their temptation and wait to eat food until after an exercise ended (Tronieri et al., 2019). Second, joint sessions may not be the ideal approach for adolescents as they tend to individuate themselves from their parents during this developmental stage, as suggested by an MBPG intervention with youth aged 12-17 with intellectual or developmental disabilities and their parents (Heifetz \& Dyson, 2017). Additionally, our results show that the effect size of joint activities showed much wider confidence intervals than non-joint activities, suggesting that there may be greater variations in studies with joint activities; what joint activities were included and how they were conducted may play a big difference.

Regarding the effects of MBPG interventions moderated by participant characteristics, our findings suggested that MBPG interventions have larger effects on the mental health of youth from mixed age groups than on school-aged children and adolescents. This might be the result of one particularly large effect study ( $d=2.667$; López-Alarcón et al., 2020) that included youth across 10 to 14 years of age. In addition, the larger effect size among school-aged children than among adolescents $(d=0.24$ vs. 0.106$)$ suggests that MBPG interventions may be more effective for mental health interventions with younger populations.

Regarding child gender, our findings showed that MBPG interventions were more effective in improving child mental health when the child group comprises mostly girls. This gender difference is consistent with a previous study that found greater improvements in female students' positive affect through a school-based MBI than male students, compared with their counterparts in control groups (Kang et al., 2018). One possible explanation is that female youth might be more engaged than males in response to mindfulness intervention (Bluth et al., 2017). Another explanation is that MBI may increase female youth's self-compassion more so than males, which is associated with improved emotional well-being (Kang et al., 2018). Future research is needed to further investigate the gender-specific pathways between mindfulness-based interventions and mental health outcomes.

\section{Limitations and Future Research}

Although our review included the current best evidence available, several limitations are worth noting. First, similar to several previous MBI reviews that have documented high study heterogeneity (Felver et al., 2016; Zenner et al., 2014), great variation exists in sample sizes (e.g., ranging from seven to 281 families), participant characteristics (e.g., mean age of youth ranging from 3.7 to 16.9 years), and structure and content of the parent and child sessions (e.g., dosage ranging from 4.5 to $24 \mathrm{~h}$ ) in our reviewed studies. We recognized the large heterogeneity across studies and addressed the diversity by applying the random effect models and reporting the range of true effects (Borenstein et al., 2009). 
Second, the majority of our reviewed studies are singlegroup pre-post studies without control groups. In our risk of bias assessments, the controlled studies overall showed low risk of bias in most domains. Our moderator analysis showed that the controlled studies reviewed yielded larger effect size than single-group studies $(d=0.327$ vs. 0.123$)$. This is in line with a previous review of mindfulness interventions for parents, which contained mostly single-group studies and found that controlled studies had larger effect size than single-group studies immediately after intervention $(g=0.44$ vs. 0.34; Burgdorf et al., 2019). Our finding calls for future MBPG studies to adopt a controlled study design and to use more rigorous blinding process of outcome assessment.

Third, several important variables, such as children's race/ ethnicity and family SES, were not analyzed as moderators because limited number of studies provided relevant information. The ratio of moderating variables to the included studies limits our interpretation of the findings. Also, in our moderator analyses, some small subgroups contained studies fewer than four (e.g., the school group in recruitment setting), which might lack the power to detect meaningful differences across subgroups (Hedges \& Pigott, 2004). There is a need for future studies to provide more detailed demographic information about their participants.

Finally, participant characteristics in the included studies might limit the interpretation of our findings. For example, existing MBPG interventions predominately have mothers joining the parent group rather than fathers, while mothers and fathers may react and respond differently to their children's emotions and may support their children's emotions in unique ways (Root \& Rubin, 2010). The important roles of both fathers and mothers in child emotional development call for increased involvement of fathers in future MBPG interventions. Also, the majority of involved studies were conducted in developed countries. Among the five studies that reported family SES, two focused on low-income families, whereas the other three studies focused on middleand upper-income communities. The majority of included studied did not report family SES information. This limits the generalizability of our findings to families in developing regions and low-income families. Given the detrimental mental health impact of family economic hardship, such as increasing parents' distress, increasing marital conflicts, causing harsh and inconsistent parenting, and impairing child emotional and behavioral development (Donnellan et al., 2013), more research evidence on developing regions and low-income families is imperative.

Supplementary Information The online version contains supplementary material available at https://doi.org/10.1007/s12671-021-01728-z.

Author Contribution QX led the process of study screening and data extraction, analyzed data, wrote the initial draft of the manuscript, and contributed to manuscript revision; XD screened studies, extracted data, assessed quality of studies, and contributed to writing the introduction section; RL screened studies, extracted data, assessed quality of studies, and contributed to manuscript revision; SL conceptualized and designed the study, wrote the initial draft of the manuscript, and contributed to manuscript revision. All authors approve the final manuscript as submitted and agree to be accountable for all aspects of the work.

Funding This project is funded by the University of Hong Kong Seed Fund for Basic Research.

\section{Declarations}

Ethical Statement The manuscript does not contain clinical studies or patient data.

Conflict of Interest The authors declare no competing interests.

Open Access This article is licensed under a Creative Commons Attribution 4.0 International License, which permits use, sharing, adaptation, distribution and reproduction in any medium or format, as long as you give appropriate credit to the original author(s) and the source, provide a link to the Creative Commons licence, and indicate if changes were made. The images or other third party material in this article are included in the article's Creative Commons licence, unless indicated otherwise in a credit line to the material. If material is not included in the article's Creative Commons licence and your intended use is not permitted by statutory regulation or exceeds the permitted use, you will need to obtain permission directly from the copyright holder. To view a copy of this licence, visit http://creativecommons.org/licenses/by/4.0/.

\section{References}

Bluth, K., Roberson, P. N. E., \& Girdler, S. S. (2017). Adolescent sex differences in response to a mindfulness intervention: A call for research. Journal of Child and Family Studies, 26(7), 1900-1914. https://doi.org/10.1007/s10826-017-0696-6

*Bögels, S., Hoogstad, B., van Dun, L., de Schutter, S., \& Restifo, K. (2008). Mindfulness training for adolescents with externalizing disorders and their parents. Behavioural and Cognitive Psychotherapy, 36, 193. https://doi.org/10.1017/S1352465808004190

Borenstein, M., Hedges, L. V., Higgins, J. P., \& Rothstein, H. R. (2009). Introduction to meta-analysis. Wiley. https://doi.org/10. $1002 / 9780470743386$

Bowen, M. (1966). The use of family theory in clinical practice. Comprehensive Psychiatry, 7(5), 345-374. https://doi.org/10.1016/ S0010-440X(66)80065-2

Burgdorf, V., Szabó, M., \& Abbott, M. J. (2019). The effect of mindfulness interventions for parents on parenting stress and youth psychological outcomes: A systematic review and meta-analysis. Frontiers in Psychology, 10, 1336. https://doi.org/10.3389/fpsyg. 2019.01336

Cohen, J. (1992). A power primer. Psychological Bulletin, 112, 155159. https://doi.org/10.1037//0033-2909.112.1.155

*de Bruin, E. I., Blom, R., Smit, F. M., van Steensel, F. J., \& Bögels, S. M. (2015). MYmind: Mindfulness training for youngsters with autism spectrum disorders and their parents. Autism, 19(8), 906914. https://doi.org/10.1177/1362361314553279

DeFrain, J., Asay, S., \& Olson, D. (2009). Family functioning. In H. T. Reis \& S. Sprecher (Eds.), Encyclopedia of human relationships 
(pp. 622-626). Sage Publications. https://doi.org/10.4135/97814 12958479.n202.

Donnellan, M. B., Martin, M. J., Conger, K. J., \& Conger, R. D. (2013). Economic distress and poverty in families. In M. A. Fine \& F. D. Fincham (Eds.), Handbook of family theories: A content-based approach (pp. 338-355). Routledge/Taylor \& Francis Group. https://doi.org/10.4324/9780203075180.ch19.

Duncan, L. G., Coatsworth, J. D., Gayles, J. G., Geier, M. H., \& Greenberg, M. T. (2015). Can mindful parenting be observed? Relations between observational ratings of mother-youth interactions and mothers' self-report of mindful parenting. Journal of Family Psychology, 29(2), 276-282. https://doi.org/10.1037/a0038857

European Commission. (1998). Minimum data set of European mental health indicators. https://ec.europa.eu/health/ph_projects/1998/ monitoring/fp_monitoring_1998_annexe2_09_en.pdf.

Felver, J. C., Celis-de Hoyos, C. E., Tezanos, K., \& Singh, N. N. (2016). A systematic review of mindfulness-based interventions for youth in school settings. Mindfulness, 7(1), 34-45. https://doi. org/10.1007/s12671-015-0389-4

Galderisi, S., Heinz, A., Kastrup, M., Beezhold, J., \& Sartorius, N. (2015). Toward a new definition of mental health. World Psychiatry, 14(2), 231-233. https://doi.org/10.1002/wps.20231

Goldberg, J. S., \& Carlson, M. J. (2014). Parents' relationship quality and children's behavior in stable married and cohabiting families. Journal of Marriage and Family, 76(4), 762-777. https://doi.org/ 10.1111/jomf.12120

Greco, L. A., Baer, R. A., \& Smith, G. T. (2011). Assessing mindfulness in children and adolescents: Development and validation of the Child and Adolescent Mindfulness Measure (CAMM). Psychological Assessment, 23(3), 606-614. https://doi.org/10.1037/ a0022819

*Hancock, K. M., Swain, J., Hainsworth, C. J., Dixon, A. L., Koo, S., $\&$ Munro, K. (2018). Acceptance and commitment therapy versus cognitive behavior therapy for children with anxiety: Outcomes of a randomized controlled trial. Journal of Clinical Child \& Adolescent Psychology, 47(2), 296-311. https://doi.org/10.1080/15374 416.2015.1110822

*Haydicky, J., Shecter, C., Wiener, J., \& Ducharme, J. M. (2015). Evaluation of MBCT for adolescents with ADHD and their parents: Impact on individual and family functioning. Journal of Child and Family Studies, 24(1), 76-94. https://doi.org/10.1007/ s10826-013-9815-1

Hedges, L. V., \& Pigott, T. D. (2004). The power of statistical tests for moderators in meta-analysis. Psychological Methods, 9(4), 426-445. https://doi.org/10.1037/1082-989X.9.4.426

*Heifetz, M., \& Dyson, A. (2017). Mindfulness-based group for teens with developmental disabilities and their parents: A pilot study. Mindfulness, 8(2), 444-453. https://doi.org/10.1007/ s12671-016-0616-7

*Jackman, M. M., Nabors, L. A., McPherson, C. L., Quaid, J. D., \& Singh, N. N. (2019). Feasibility, acceptability, and preliminary effectiveness of the OpenMind (OM) program for pre-school children. Journal of Child and Family Studies, 28(10), 2910-2921. https://doi.org/10.1007/s10826-019-01506-5

Jefferson, F. A., Shires, A., \& McAloon, J. (2020). Parenting selfcompassion: A systematic review and meta-analysis. Mindfulness, 11, 2067-2088. https://doi.org/10.1007/s12671-020-01401-x

Kabat-Zinn, M., \& Kabat-Zinn, J. (1997). Everyday blessings The inner work of mindful parenting. Hyperion.

Kang, Y., Rahrig, H., Eichel, K., Niles, H. F., Rocha, T., Lepp, N. E., Gold, J., \& Britton, W. B. (2018). Gender differences in response to a school-based mindfulness training intervention for early adolescents. Journal of School Psychology, 68, 163-176. https://doi. org/10.1016/j.jsp.2018.03.004

Kim, S. Y., Park, J. E., Lee, Y. J., Seo, H. J., Sheen, S. S., Hahn, S., Jang, B. H., \& Son, H. J. (2013). Testing a tool for assessing the risk of bias for nonrandomized studies showed moderate reliability and promising validity. Journal of Clinical Epidemiology, 66(4), 408-414. https://doi.org/10.1016/j.jclinepi.2012.09.016

Kostova, Z., Levin, L., Lorberg, B., \& Ziedonis, D. (2019). Mindfulness-based interventions for adolescents with mental health conditions: A systematic review of the research literature. Journal of Child and Family Studies, 28(10), 2633-2649. https://doi.org/ 10.1007/s10826-019-01477-7

*Kumar, S., Croghan, I. T., Biggs, B. K., Croghan, K., Prissel, R., Fuehrer, D., Donelan-Dunlap, B., \& Sood, A. (2018). Familybased mindful eating intervention in adolescents with obesity: A pilot randomized clinical trial. Children, 5(7), 93. https://doi.org/ 10.3390/children5070093

Lipsey, M. W., \& Wilson, D. B. (2001). Practical meta-analysis. SAGE publications.

Lohaus, A., Chodura, S., Möller, C., Symanzik, T., Ehrenberg, D., Job, A. K., Reindl, V., Konrad, K., \& Heinrichs, N. (2017). Children's mental health problems and their relation to parental stress in foster mothers and fathers. Child and Adolescent Psychiatry and Mental Health, 11, 43. https://doi.org/10.1186/ s13034-017-0180-5

*Lo, H. H., Wong, S. W., Wong, J. Y., Yeung, J. W., Snel, E., \& Wong, S. Y. (2017). The effects of family-based mindfulness intervention on ADHD symptomology in young children and their parents: A randomized control trial. Journal of Attention Disorders, 24(5), 667-680. https://doi.org/10.1177/1087054717743330

*Lo, H. H., Wong, J. Y., Wong, S. W., Wong, S. Y., Choi, C. W., Ho, R. T., Fong, R. W., \& Snel, E. (2019). Applying mindfulness to benefit economically disadvantaged families: A randomized controlled trial. Research on Social Work Practice, 29(7), 753-765. https://doi.org/10.1177/1049731518817142

*López-Alarcón, M., Zurita-Cruz, J. N., Torres-Rodríguez, A., BediaMejía, K., Pérez-Güemez, M., Jaramillo-Villanueva, L., RendónMacías, M. E., Fernández, J. R., \& Martínez-Maroñas, P. (2020). Mindfulness affects stress, ghrelin, and BMI of obese children: A clinical trial. Endocrine Connections, 9(2), 163-172. https://doi. org/10.1530/EC-19-0461

*Martin, S., Wolters, P. L., Toledo-Tamula, M. A., Schmitt, S. N., Baldwin, A., Starosta, A., Gillespie, A., \& Widemann, B. (2016). Acceptance and commitment therapy in youth with neurofibromatosis type 1 (NF1) and chronic pain and their parents: A pilot study of feasibility and preliminary efficacy. American Journal of Medical Genetics Part A, 170(6), 1462-1470. https://doi.org/ 10.1002/ajmg.a.37623

McInnes, M., Moher, D., Thombs, B., McGrath, T., Bossuyt, P., \& the PRISMA-DTA Group. (2018). Preferred reporting items for a systematic review and meta-analysis of diagnostic test accuracy studies. JAMA, 319(4), 388-396. https://doi.org/10.1001/jama. 2017.19163

Manning, C., \& Gregoire, A. (2006). Effects of parental mental illness on children. Psychiatry, 5(1), 10-12. https://doi.org/10.1383/psyt. 2006.5.1.10

Neece, C. L. (2014). Mindfulness-based stress reduction for parents of young children with developmental delays: Implications for parental mental health and child behavior problems. Journal of Applied Research in Intellectual Disabilities, 27(2), 174-186. https://doi. org/10.1111/jar.12064

Potharst, E. S., Baartmans, J. M., \& Bögels, S. M. (2021). Mindful parenting training in a clinical versus non-clinical setting: An explorative study. Mindfulness, 12, 504-518. https://doi.org/10. 1007/s12671-018-1021-1

*Racey, D. N., Fox, J., Berry, V. L., Blockley, K. V., Longridge, R. A., Simmons, J. L., Janssens, A., Kuyken, W., \& Ford, T. J. (2018). Mindfulness-based cognitive therapy for young people and their carers: A mixed-method feasibility study. Mindfulness, 9(4), 1063-1075. https://doi.org/10.1007/s12671-017-0842-7 
Ridderinkhof, A., de Bruin, E. I., Blom, R., \& Bögels, S. M. (2018). Mindfulness-based program for children with autism spectrum disorder and their parents: Direct and long-term improvements. Mindfulness, 9(3), 773-791. https://doi.org/10.1007/ s12671-017-0815-x

Root, A. K., \& Rubin, K. H. (2010). Gender and parents' reactions to children's emotion during the preschool years. New Directions for Child and Adolescent Development, 128, 51-64. https://doi. org/10.1002/cd.268

*Salem-Guirgis, S., Albaum, C., Tablon, P., Riosa, P. B., Nicholas, D. B., Drmic, I. E., \& Weiss, J. A. (2019). MYmind: A concurrent group-based mindfulness intervention for youth with autism and their parents. Mindfulness, 10(9), 1730-1743. https://doi.org/10. 1007/s12671-019-01107-9

*Shaffer, R. C., Wink, L. K., Ruberg, J., Pittenger, A., Adams, R., Sorter, M., Manning, P., \& Erickson, C. A. (2019). Emotion regulation intensive outpatient programming: Development, feasibility, and acceptability. Journal of Autism and Developmental Disorders, 49(2), 495-508. https://doi.org/10.1007/s10803-018-3727-2

Shorey, S., \& Ng, E. D. (2021). The efficacy of mindful parenting interventions: A systematic review and meta-analysis. International Journal of Nursing Studies, 121, 103996. https://doi.org/ 10.1016/j.ijnurstu.2021.103996

Sterne, J. A., Egger, M., \& Smith, G. D. (2001). Investigating and dealing with publication and other biases in meta-analysis. BMJ, 323, 101-105. https://doi.org/10.1136/bmj.323.7304.101

Townshend, K., Jordan, Z., Stephenson, M., \& Tsey, K. (2016). The effectiveness of mindful parenting programs in promoting parents' and children's wellbeing: A systematic review. JBI Database of Systematic Reviews and Implementation Reports, 14(3), 139-180. https://doi.org/10.11124/JBISRIR-2016-2314.

*Tronieri, J. S., Wadden, T. A., Leonard, S. M., \& Berkowitz, R. I. (2019). A pilot study of acceptance-based behavioural weight loss for adolescents with obesity. Behavioural and Cognitive Psychotherapy, 47(6), 686-696. https://doi.org/10.1017/S1352 465819000262

United Nations Development Programme. (2019). Human development report 2019. http://hdr.undp.org/en/2019-report.

*Van der Oord, S., Bögels, S. M., \& Peijnenburg, D. (2012). The effectiveness of mindfulness training for children with ADHD and mindful parenting for their parents. Journal of Child and Family Studies, 21(1), 139-147. https://doi.org/10.1007/ s10826-011-9457-0
Van de Weijer-Bergsma, E., Formsma, A. R., de Bruin, E. I., \& Bögels, S. M. (2012). The effectiveness of mindfulness training on behavioral problems and attentional functioning in adolescents with ADHD. Journal of Child and Family Studies, 21(5), 775-787. https://doi.org/10.1007/s10826-011-9531-7

Verhagen, A. P., de Vet, H. C., de Bie, R. A., Kessels, A. G., Boers, M., Bouter, L. M., \& Knipschild, P. G. (1998). The Delphi list: A criteria list for quality assessment of randomized clinical trials for conducting systematic reviews developed by Delphi consensus. Journal of Clinical Epidemiology, 51(12), 1235-1241. https:// doi.org/10.1016/s0895-4356(98)00131-0

Wang, L., \& Crane, D. R. (2001). The relationship between marital satisfaction, marital stability, nuclear family triangulation, and childhood depression. American Journal of Family Therapy, 29(4), 337-347. https://doi.org/10.1080/01926180126502

Wang, Q., \& Zhou, T. (2015). The impact of family functioning and mental health condition on the child's behavioral problems. Social Behavior and Personality: An International Journal, 43(7), 11351146. https://doi.org/10.2224/sbp.2015.43.7.1135

World Health Organization. (WHO). (2013). Comprehensive mental health action plan 2013-2020. https://apps.who.int/gb/ebwha/ pdf_files/WHA66/A66_R8-en.pdf.

Xie, Q-W., Lu, S., Dai, X., \& Lyu, R. (2020). Effects of mindfulnessbased parallel parent-child group interventions: A systematic review. PROSPERO 2020 CRD42020164927. https://www.crd. york.ac.uk/prospero/display_record.php?RecordID=164927.

Zenner, C., Herrnleben-Kurz, S., \& Walach, H. (2014). Mindfulnessbased interventions in schools - a systematic review and metaanalysis. Frontiers in Psychology, 5, 603. https://doi.org/10.3389/ fpsyg.2014.00603

*Zhang, D., Chan, S. K. C., Lo, H. H. M., Chan, C. Y. H., Chan, J. C. Y., Ting, K. T., Gao, T. T., Lai, K. Y., Bögels, S. M., \& Wong, S. Y. S. (2017). Mindfulness-based intervention for Chinese children with ADHD and their parents: A pilot mixed-method study. Mindfulness, 8, 859-872. https://doi.org/10.1007/s12671-016-0660-3

Publisher's Note Springer Nature remains neutral with regard to jurisdictional claims in published maps and institutional affiliations. 